\title{
Synthesis and quantitative comparison of polymer-coated quantum dots with integrated acceptor dyes as FRET-based nanoprobes
}

María Theresa Fernández-Argüelles, Aleksey Yakovlev, Ralph A. Sperling, Camilla Luccardini, Stéphane Gaillard, Alfredo Sanz Medel, Jean-Maurice Mallet, Jean-Claude Brochon, Anne Feltz, Martin Oheim, and Wolfgang J. Parak

\section{Supporting Information ${ }^{1}$}

\section{Synthesis of the amphiphilic polymer}

I.1. Synthesis of plain amphiphilic polymer without incorporated dye (PMA75C12)

I.2. Synthesis of ATTO-dye-modified amphiphilic polymer

II. Polymer-coating of quantum dots

II.1. Overview of synthesized configurations

II.2. Polymer-coating of quantum dots with polymer with / without dye

II.3. Synthesis of empty micelles as control

II.4. Attachment of ATTO dye to polymer-coated quantum dots with EDC

II.5. PEG-modification of polymer-coated quantum dots with subsequent ATTO modification

\section{Sample purification with gel electrophoresis}

III.1. ATTO dye

III.2. Polymer-coated quantum dots with incorporated ATTO dye

III.3. Polymer-coated quantum dots with ATTO dye attached in post modification

III.4. Polymer-coated quantum dots with ATTO dye attached to a shell of PEG

$\underline{\text { IV. Normalization procedure for the evaluation of fluorescence spectra }}$

V. Evaluation of the fluorescence spectra

V.1. Polymer-coated quantum dots with incorporated ATTO dye

V.2. Polymer-coated quantum dots with ATTO dye attached in post modification

V.3. Polymer-coated quantum dots with ATTO dye attached to a shell of PEG

VI. Time resolved measurements

V.1. Experimental set-up

V.2. Polymer-coated quantum dots with incorporated ATTO dye

V.3. Polymer-coated quantum dots with ATTO dye attached in post modification

V.4. Polymer-coated quantum dots with ATTO dye attached to a shell of PEG

\section{VII: References}

\footnotetext{
${ }^{1}$ The idea of this Supporting Information is to provide the interested reader with a detailed account of the raw data measured. It is by far not meant to be read from the beginning until the end. In contrast, due to the index on this page the reader can access the piece of information that he/she is interested in. All important and relevant information is included in the main paper and this Supporting Information expands on further details to specific points. We have taken great care to describe here everything as detailed as possible, so that a documentation about all experimental and evaluation steps is given.
} 


\section{Synthesis of the amphiphilic polymer}

\section{I.1. Synthesis of plain amphiphilic polymer without incorporated dye (PMA75C12) $^{2}$}

Poly(isobutylene-alt-maleic anhydride) is designed to form the hydrophilic backbone on the amphiphilic polymer. Hydrophobic side chains are attached by reacting the aminogroups of dodecylamine (C12 carbon chain) with the anhydride rings of the hydrophilic backbone in order to generate an amphiphilic polymer. The mixture is chosen so that $75 \%$ of the available anhydride rings are going to react with the amino-group of dodecylamine. Afterwards, the polymer still has $25 \%$ anhydride rings that can react with cross-linker or ATTO-dye.

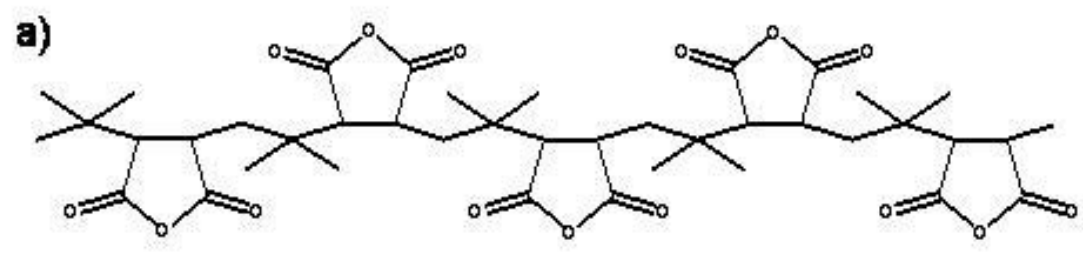

c)

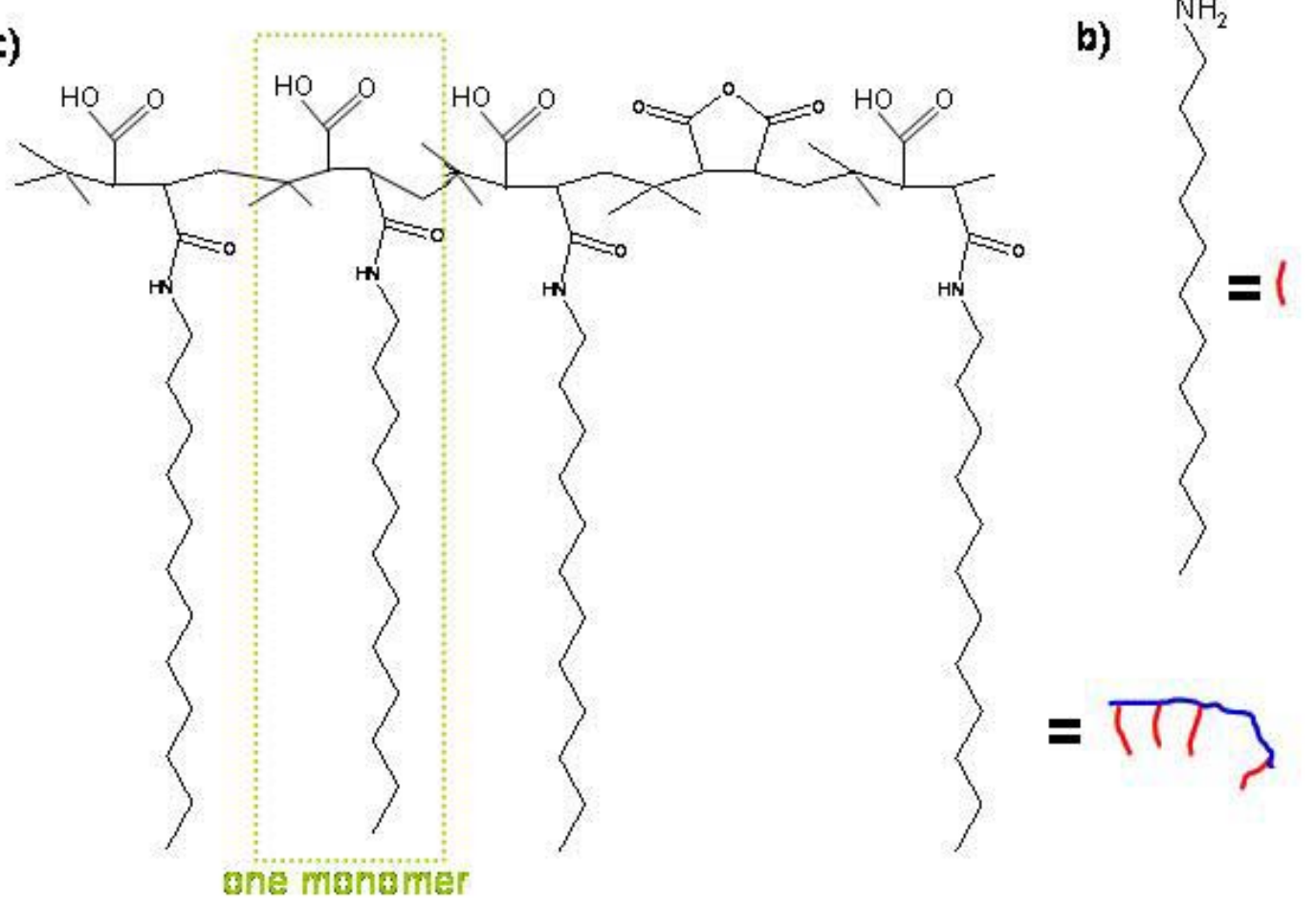

Figure SI-I.1: Structure of the amphiphilic polymer. a) The hydrophilic backbone is made out of poly(isobutylene-alt-maleic anhydride). Each monomer unit comprises one anhydride ring. The hydrophilic nature is symbolized by blue color. b) Dodecylamine is used to provide hydrophobic side chains. The hdyrophobic nature is symbolized by red color. c) Upon reaction of the amino group of dodecylamine to anhydride rings of the polymer backbone an amphiphilic polymer with a hydrophilic backbone and hydrophobic sidechains is created. The stoichiometry has been chosen in a way that $75 \%$ of the anhydride rings are reacted with dodecylamine and $25 \%$ of the anhydride rings remain intact.

\footnotetext{
${ }^{2}$ The synthesis of the amphiphilic polymer has been invented by Chen-An J. Lin and will be reported in more detail elsewhere (Ref $\# 1$ ). This polymer refers to substance $\mathrm{D}_{0}$ in Figure SI-II.1.
} 
For the synthesis of the amphiphilic polymer (in the following also called PMA75C12) $3.084 \mathrm{~g}$ (correspondent to $20 \mathrm{mmol}$ of monomer units ${ }^{3}$ ) of poly(isobutylene-alt-maleic anhydride) (average $\mathrm{M}_{\mathrm{w}} \sim 6,000 \mathrm{~g} / \mathrm{mol}, 12-200$ mesh (85\%), Sigma, \#531278) were placed in a round flask. All the solvents employed for the synthesis of this polymer were anhydrous, in order to avoid the presence of water in the medium, which would have caused subsequent opening of the anhydride rings. In another flask, $2.70 \mathrm{~g}$ (15 mmol) of dodecylamine (DoCA, 98\%, Sigma, \# D22,220-8) were dissolved in $100 \mathrm{~mL}$ of tetrahydrofurane anhydrous (THF, $\geq 99.9 \%$, Aldrich, \#186562). The solution was added as quickly as possible under stirring to the polymer powder. This mixture was sonicated for several seconds $(\sim 20 \mathrm{~s})$, and heated to $55-60{ }^{\circ} \mathrm{C}$ (just below boiling point of THF) for 1 hour. Afterwards, the solution was pre-concentrated to about one third of the original volume in a rotation-evaporation (rotavap) system (Laborota 4000, Heidolph) under reduced pressure $(p=200-150$ mbar) in order to enhance the reaction between the polymer and the amine. Once the sample had been concentrated, it was left stirring overnight. Then, the solvent was slowly evaporated by using the rotavap system until the polymer was completely dried (pale yellow solid). Finally, the amphiphilic polymer was re-dissolved in $25 \mathrm{~mL}$ anhydrous $\mathrm{CHCl}_{3}$ with a resultant concentration of $0.8 \mathrm{M}$ monomers (=20 mmol / $25 \mathrm{~mL})$, see Figure SI-I.1.

\section{I.2. Synthesis of ATTO-dye-modified amphiphilic polymer ${ }^{4}$}

In the plain amphiphilic polymer $75 \%$ of the anhydride rings are reacted with

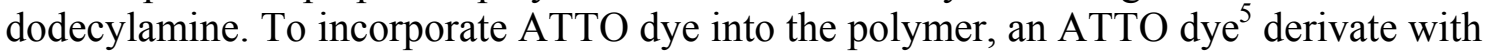
amino-group was used. By reaction of the amino-group with the remaining anhydride rings the ATTO dye is linked to the amphiphilic polymer. In the standard (1x ATTO dye) configuration $1 \%$ of the anhydride rings were reacted with ATTO, so that in total $75 \%$ of the anhydride rings serve to link dodecylamine and $1 \%$ of the anhydride rings serve to link ATTO dye.

For this purpose, in a vial, $0.5 \mathrm{mg}$ of ATTO 590-amin (ATTO-TEC GmbH, Germany) ${ }^{6}$ were dissolved in $20 \mathrm{~mL}$ of chloroform. The ATTO concentration was obtained by measuring the absorption spectra of the ATTO 590-amin by using the extinction coefficient at the absorption maximum given by the company of $120000 \mathrm{M}^{-1} \mathrm{~cm}^{-1}$. The resultant ATTO dye concentration was determined to be $9.75 \mu \mathrm{M}$. In another vial, 10 $\mu \mathrm{L}$ of the plain amphiphilic polymer PMA75C12 (0.8 M monomer unit concentration) were mixed with $8.2 \mathrm{~mL}$ of ATTO 590 -amin $9.75 \mu \mathrm{M}$ in anhydrous chloroform. Thus, the amount of added dye is $1 \%$ of the total amount of monomers. The mixture was stirred at room temperature overnight in order to allow the reaction between the anhydride rings of the polymer with the amino groups of the ATTO 590 dye. The chloroform was evaporated and the resulting powder dissolved in $10 \mu \mathrm{L}$ of anhydrous chloroform to yield a resultant concentration of $0.8 \mathrm{M}$ monomers.

\footnotetext{
${ }^{3}$ One polymer unit $\left\langle\mathrm{M}_{\mathrm{w}}>\sim 6,000 \mathrm{~g} / \mathrm{mol}\right.$ consists of ca. 39 monomers. The molecular weight of each monomer is $\mathrm{M}_{\mathrm{w}}=154 \mathrm{~g} / \mathrm{mol} \Rightarrow 3.084 \mathrm{~g} / 154 \mathrm{gmol}^{-1}=20 \mathrm{mmol}$.

${ }^{4}$ This polymer refers to substance $\mathrm{D}_{1}$ in Figure SI-II.1.

5 emission maxima at $620 \mathrm{~nm}$, absorption maximum: $596 \mathrm{~nm}$.

${ }^{6}$ This dye has a similar structure as ATTO 590-COOH, \#AD 590-2, but with an amino group instead of the carboxylic acid
} 


\section{Polymer-coating of quantum dots}

\section{II.1. Overview of synthesized configurations}

For the here presented study a variety of different samples was prepared. An overview of these samples is presented in Figure SI-II.1.

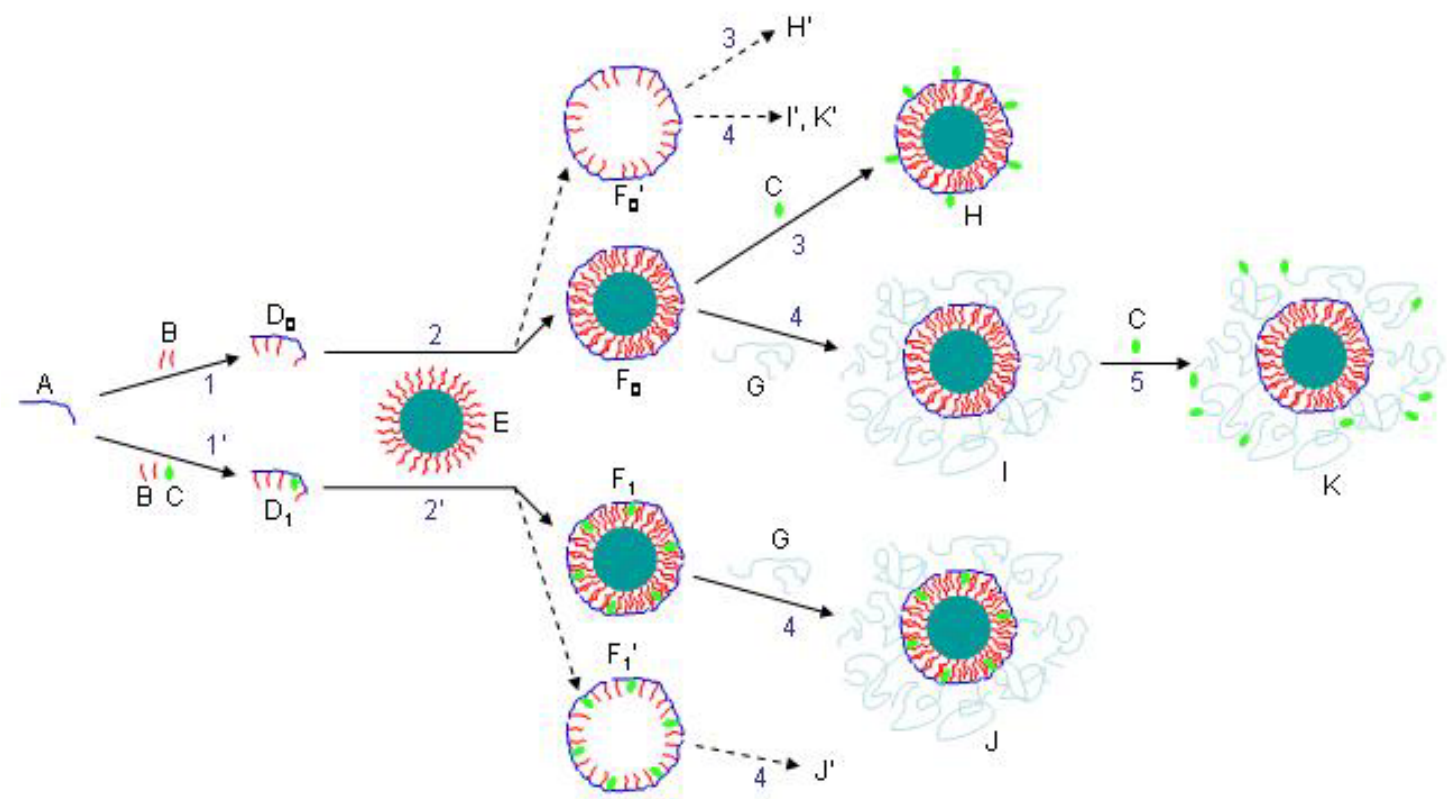

Fiqure SI-II.1: A hydrophilic polymer backbone (A) is modified with hydrophobic sidechains (B) and optionally with ATTO dye $(C)$ to yield an amphiphilic polymer with $\left(D_{I}\right)$ and without $\left(D_{0}\right)$ incorporated ATTO dye. Surfactant stabilized hydrophobic quantum dots (E) can be transferred into aqueous solution by wrapping the amphiphilic polymer $(D)$ around them. Besides polymer-coated quantum dots $\left(F_{0}, F_{1}\right)$ also empty micelles $\left(F_{0}{ }^{\prime}, F_{1}{ }^{\prime}\right)$ result from this reaction as by-product. ATTO-dye $(C)$ can be attached to the polymer-coated particles $\left(F_{0}\right)$ in a post reaction using EDC chemistry to form polymer-coated quantum dots with dye linked to their shell $(H)$. The polymer-coated quantum dots $\left(F_{0}, F_{1}\right)$ can also be modified by attachment of polyethyleneglycol $(P E G, G)$ to yield particles with very high colloidal stability (I, J). ATTO-dye can also be reacted to the PEG shell, whereby in the resulting product (K) the ATTO dye is farther away from the quantum dot surface than in the other configurations.

\section{II.2. Polymer-coating of quantum dots with polymer with / without dye ${ }^{7}$}

Polymer-coating of quantum dots was performed according to previously published protocols $^{2,3}$. In the here described experiments, the optimum number of added polymer monomer units per $\mathrm{nm}^{2}$ of nanoparticle surface was 70 , and the amount of cross-linker molecules was $5 \%$ of the total number of added polymer monomers (i.e. per anhydride ring).

$15 \mu \mathrm{L}$ of PMA75C12 with and without incorporated ATTO dye (0.8 M monomer concentration, see Chapter I) were placed in a round flask with $\sim 1 \mathrm{~mL}$ of anhydrous chloroform and $800 \mu \mathrm{L}$ of a $2.7 \mu \mathrm{M}$ solution of CdSe/ZnS quantum dots (Qdot 545 ITK

\footnotetext{
${ }^{7}$ These quantum dots refer to particles $\mathrm{F}_{1}$ and $\mathrm{F}_{0}$ in Figure SI-II.1.
} 
organic quantum dots ${ }^{8}$, Invitrogen, \#Q21791MP) in anhydrous chloroform, respectively 9 . The flask was heated to $55-60{ }^{\circ} \mathrm{C}$ for approximately $40 \mathrm{~s}$ in order to give more flexibility to the polymer chain, and to allow for a better coating. Then the mixture was stirred in a rotavap system for $\sim 15 \mathrm{~min}$. The solvent was removed by reduced pressure at $200 \mathrm{mbar}$, and when there was no more solvent the pressure was decreased down to 20 mbar in order to dry the sample completely. Afterwards, $30 \mu \mathrm{L}$ of $0.02 \mathrm{M}$ bis(hexamethylene)triamine (Fluka, \#14506) in chloroform were added to the dried powder, and the mixture was stirred for $\sim 15 \mathrm{~min}$. In this way, the amount of added cross-linker is $5 \%$ of the total amount of monomers ${ }^{10}$. However, the cross-linker possesses two amino groups, so finally $10 \%$ of the monomer units will react with the amino groups. The chloroform was evaporated with a rotavap system at $p=200$ mbar. When the mixture was dry, the pressure was further decreased down to 20 mbar to dry the sample completely. The powder of polymer coated quantum dots was redissolved in sodium borate buffer ${ }^{11}$ (SBB, $50 \mathrm{mM}$ sodium borate, $\mathrm{pH}=12$. For purification the samples were filtrated through a centrifuge filter device with $0.22 \mu \mathrm{m}$ pore size (Ultrafree-CL GV Filter, Millipore, \#UFC40GV25) at the speed of $3500 \mathrm{rpm}$ for 5 minutes. Then, the samples were concentrated in a 100000 MWCO centrifuge filter (Amicon Ultra-4 Centrifugal Filter Units, Millipore, \# UFC810096), and the buffer was exchanged to SBB pH 9 by adding this buffer 3 times and concentrating again until to get a final volume of $\sim 1 \mathrm{~mL}$.

In addition to these standard polymer-coated quantum dots also quantum dots with a different polymer / quantum dot ratio were produced. The synthesis procedure was exactly the same, besides the different amount of added polymer. X-times polymer coated quantum dots refer to quantum dots that have been transferred to water with $\mathrm{X}$ times of the amount of polymer as used in the standard procedure ${ }^{12}$. The crosslinker / monomer ratio was always adjusted to $5 \%$.

\footnotetext{
${ }^{8}$ The quantum dots dissolved in decane were first precipitated with methanol and then redissolved in chloroform. We determined the effective diameter of the quantum dots in the following way: according to a previous study by another group the wavelength of the first exciton peak in the absorption spectrum of the CdSe/ZnS quantum dots of $527 \mathrm{~nm}$ corresponds to a diameter of the CdSe core of $2.65 \mathrm{~nm}$ (neglecting the shift in the absorption peak due to the $\mathrm{ZnS}$ shell) (see Ref. \#4). In order to take into account the $\mathrm{ZnS}$ shell and the TOPO coating $2 \cdot 0.2 \mathrm{~nm}$ and $2 \cdot 1.0 \mathrm{~nm}$ were added, respectively. This leads to an effective diameter of $5.05 \mathrm{~nm}$ for one TOPO-coated $\mathrm{CdSe} / \mathrm{ZnS}$ quantum dot. Thus, the surface of 1 quantum dot is $4 \pi \cdot(5.05 \mathrm{~nm} / 2)^{2}=80.1 \mathrm{~nm}^{2}$. After the polymer-coating the resultant surface area in aqueous solution will be significantly bigger (see Ref. \#5).

${ }^{9}(15 \mu \mathrm{L} \cdot 0.8 \mathrm{M}) /(800 \mu \mathrm{L} \cdot 2.7 \mu \mathrm{M})=5600 \Rightarrow$ ca. 5600 polymer monomer units are added per quantum dot;

$\Rightarrow 5600$ polymer units $/ 80.1 \mathrm{~nm}^{2}=70$ polymer monomer units per $\mathrm{nm}^{2}$ nanoparticle surface.

In the case when $1 \%, 2 \% ; 4 \%$, and $8 \%$ of the monomer units were modified with ATTO dye in total 56 , 112,224 , and 448 ATTO dye molecules were added per quantum dot. This has to be considered as upper limit, as some of the added polymer (with the attached dye) did not wrap around the particles but formed empty micelles.

${ }^{10}(30 \mu \mathrm{L} \cdot 0.02 \mathrm{M}) /(15 \mu \mathrm{L} \cdot 0.8 \mathrm{M})=0,05=5 \%$

${ }^{11}$ For the preparation of 2.51 of SBB, $250 \mathrm{~mL}$ of $0.5 \mathrm{M}$ boric acid (Sigma, \#B0252) was mixed with $2 \mathrm{~L}$ MilliQ water, and the $\mathrm{pH}$ was adjusted by adding the appropriate amount of $1 \mathrm{M}$ sodium hydroxide (Roth, \#KO21.2). Finally the volume was adjusted with water to $2.5 \mathrm{~L}$.

${ }^{12}$ I.e. $70 \cdot \mathrm{X}$ polymer monomer units were added per $\mathrm{nm}^{2}$ quantum dot surface.
} 


\section{II.3. Synthesis of empty micelles as control ${ }^{13}$}

For the preparation of empty polymer micelles as control samples the same procedure as described in Chapter II.2 was carried out, but with plain chloroform instead of a solution of quantum dots in chloroform. As no quantum dots are present in solution the resultant particles can be ascribed to "empty" micelles of the polymer.

\section{II.4. Attachment of ATTO dye to polymer-coated quantum dots with EDC ${ }^{14}$}

For the preparation of these samples, first a batch of water-soluble polymer coated quantum dots (without dye in the polymer-shell) was prepared according to Chapter II.2. Amino-modified ATTO-dye was then attached to the outer polymer shell via EDC chemistry.

Polymer coated quantum dots (with no dye in the polymer-shell) were incubated with the appropriate amount of amino-modified ATTO-dye dissolved in SBB $\mathrm{pH} 9$ and freshly prepared EDC (N-(3-Dimethylaminopropyl)-N'-ethylcarbodiimide hydrochloride) (Sigma-Aldrich \#E7750). The mixture was stirred for two hours, and then run in gel electrophoresis. Details for linking molecules with amino-groups to the polymer-shell with EDC have been published in a previous report ${ }^{3}$.

So as to evaluate the necessary amount of EDC needed for the linkage, first a series of quantum dots was made in which the ATTO-dye to quantum dot ratio was kept constant to a value of 40 . Within the series the EDC to quantum dot ratio was varied between 0 and 128000 and the resulting conjugates were analyzed by gel electrophoresis. The value of 2000 for the EDC / quantum dot ratio was found appropriate and this ratio was used for all further experiments.

For the following experiments the ATTO-dye to quantum dot ratio was varied between $5 \times 10^{-3}$ and 320 by mixing the appropriate amounts of amino-modified ATTO-dye to polymer coated quantum dots, whereby the EDC to quantum dot ratio was kept constant to 2000 .

\section{II.5. PEG-modification of polymer-coated quantum dots with subsequent ATTO $\underline{\text { modification }^{15}}$}

In order to evaluate the effect of the distance between ATTO-dye and quantum dot surface the dye was also attached onto the external side of a PEG shell around the quantum dots. We here assume that the distribution average over all acceptor molecules attached to the central quantum dot and the relative donor-acceptor dipole orientation is unchanged for different distance-configurations over time. For the preparation of these samples, polymer coated quantum dots (see Chapter II.2) had to be first modified with poly(ethyleneglycol) (PEG). This was done by using diamino-PEG $\left(\mathrm{M}_{\mathrm{W}}=2 \mathrm{kDa}, \mathrm{NH}_{2}-\right.$ PEG-NH ${ }_{2}$, Rapp Polymere, Germany, \# 112000-2) and EDC (Sigma-Aldrich \# E7750) according to a previously published protocol ${ }^{3}$.

\footnotetext{
${ }^{13}$ These polymer micelles refer to particles $\mathrm{F}_{1}{ }^{\prime}$ and $\mathrm{F}_{0}{ }^{\prime}$ in Figure SI-II.1.

14 This refers to particles $\mathrm{H}$ in Figure SI-II.1.

${ }^{15}$ This refers to particles I and K in Figure SI-II.1.
} 
In a first experiment the necessary amount of PEG and EDC to saturate the surface of the polymer-coated quantum dots with PEG chains had to be determined. In order to carry out this experiment, polymer coated quantum dots were placed in a vial and mixed with the appropriate amount of PEG in order to get a PEG / quantum dot excess ratio of 500 (this value is based on previous studies). Then a series of samples with increasing amounts of added EDC was prepared, whereby the EDC / quantum dot ratio was varied from 0 to $2 \times 10^{6}$. After two hours of reaction, the mixtures were run with gel electrophoresis, see Figure SI-II.2. From these results the value 2000 for the EDC / quantum dot ratio was selected for all further experiments.

Then, a large batch of polymer-coated quantum dots saturated with PEG on their surface was synthesized as described above with the following values: PEG / quantum dot $=$ $500, \mathrm{EDC} /$ quantum dot $=2000$. To these samples NHS-functionalized ATTO dye was added. Via the NHS-group the ATTO dye was directly attached to the amino-groups of the ends of the PEG that are pointing towards solution. For our experiments the NHSATTO / quantum dot ratio was varied between 0 and 1655 .

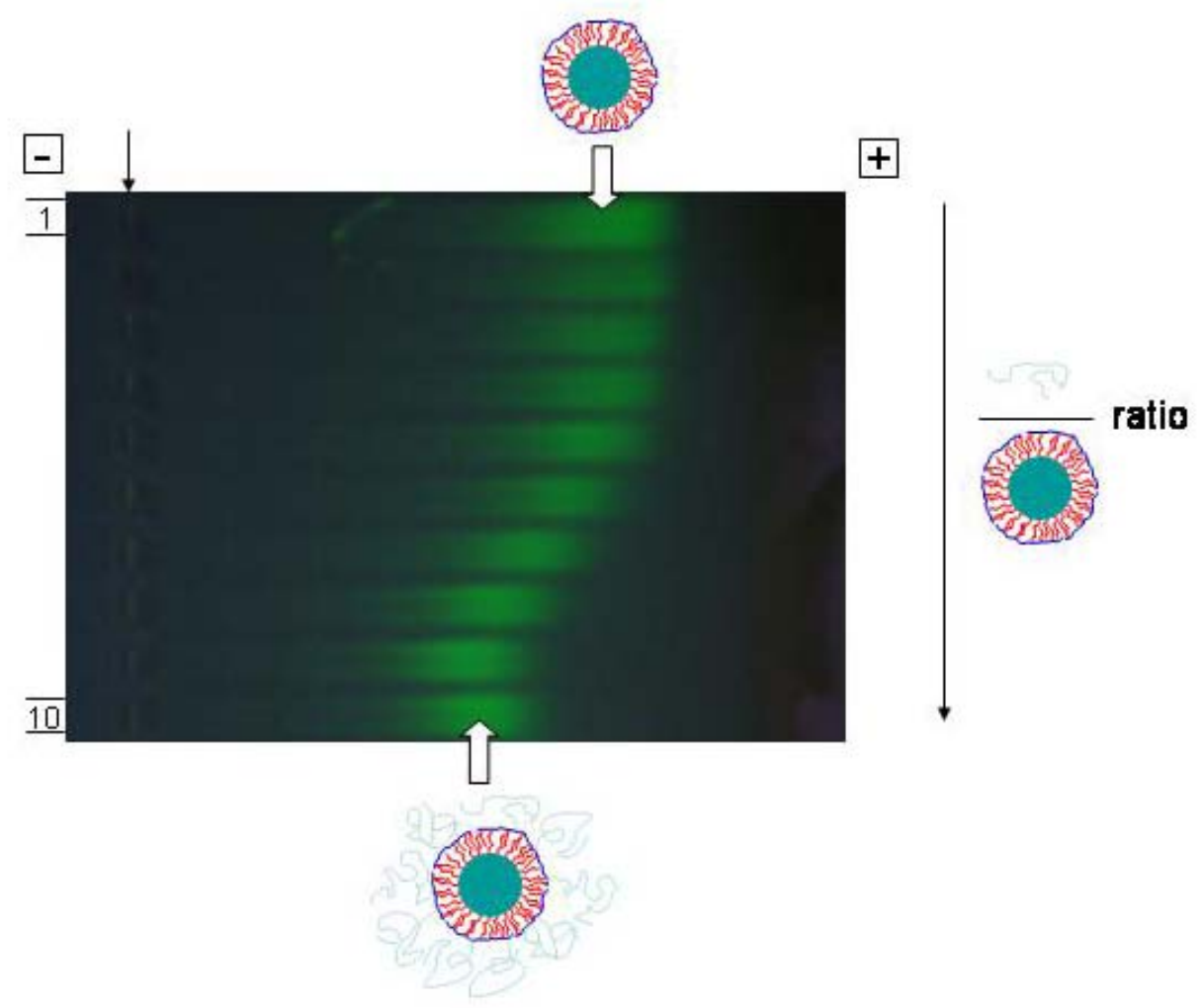

Figure SI-II.2. Diamino-PEG has been attached to polymer-coated quantum dots. While the PEG / quantum dot ratio is kept constant at 500, the EDC / quantum dot ratio is gradually increased from the sample shown in lane 1 to the sample shown in lane 10. These samples were run on an $1 \%$ agarose gel and the position of the samples was imaged with a digital camera upon UV-illumination of the gel. The more EDC is added to the PEG / quantum dot mixture, the more PEG is attached per quantum dot, the bigger the conjugates become, and the slower they migrate on the gel. At one point the addition of more EDC does not lead to more attachment of PEG, as the quantum dot surface is already saturated with PEG. This EDC / quantum dot ratio is selected for the following experiments to warrant for a saturation of the quantum dot surface with PEG. Too much added EDC on the other hand would facilitate particle agglomeration. 


\section{Sample purification with gel electrophoresis}

Gel electrophoresis was performed in Agarose UltraPure (Invitrogen \#15510027) 1\%, in 0.5xTBE buffer (Sigma-Aldrich, \# T3913). After running the samples for 1 hour at 100 V (Powerpac, Bio-Rad), a picture was recorded with a digital camera (Canon Digital Ixus IIs) under the irradiation of UV light.

\section{III.1. ATTO dye}

To investigate the electrophoretic mobility of free ATTO dye as comparison to the mobilities of quantum dots, both ATTO-dye modifications, the amino- and the NHSmodified ATTO, were run on a $1 \%$ agarose gel at $100 \mathrm{~V}$ in TBE buffer (TRIS-BorateEDTA, $\mathrm{pH}=8-9)$. The resultant gel is shown in Figure SI-III.1. This image demonstrates, that in alkaline conditions the amino-modified ATTO dye is positively charged due to the protonation of the amine. This is why this molecule migrates towards the minus pole. For the NHS-modified ATTO dye three bands could be detected. The molecules of the first fraction do not posses any charge and therefore do not move. The molecules of the second and third fraction are negatively charged and therefore migrate towards the plus pole. We speculate that the second and third fraction might be associated to molecules with hydrolized NHS-group.

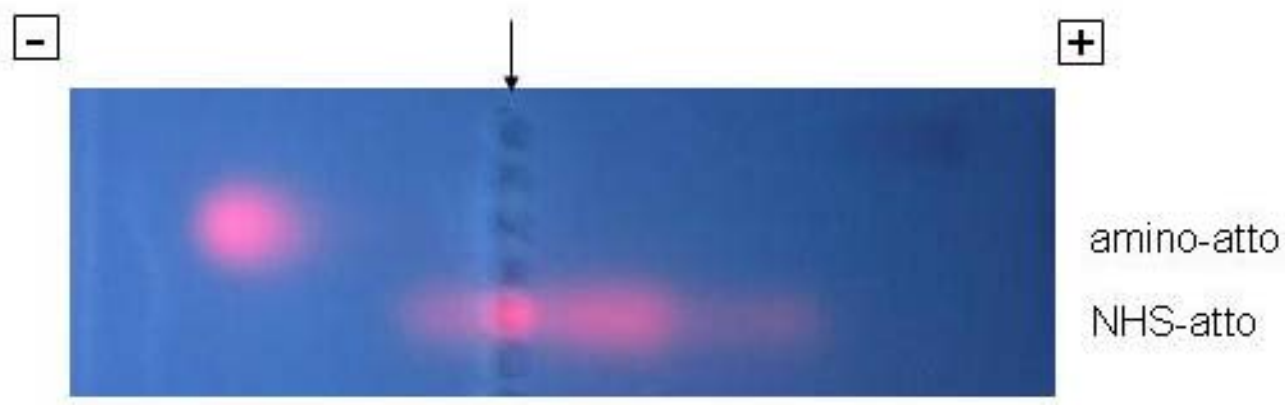

Fiqure SI-III.1: Image of a 1\% agarose gel under UV-illumination after running samples of amino- and NHS-modified ATTO dye at 100V. The arrow indicates the position where the samples had been loaded.

\section{III.2. Polymer-coated quantum dots with incorporated ATTO dye}

On Figures SI-III.2, SI-III.3, and SI-III.4 the images of gels are shown, in which different samples of polymer-coated quantum dots have been run. Due to the negative charge of the polymer-shell all samples migrate towards the plus pole. In the first lanes of all the three gels a sample of micelles formed from the polymer with embedded dye ( $\mathrm{F}_{1}{ }^{\prime}$ from Figure SI-II.1, see also Chapter II.3) was run ${ }^{16}$. This demonstrates clearly, that the amphiphilic polymer forms micelles. Single remaining polymer molecules had been washed away during the purification process with the centrifuge filters. However, the micelles formed by the polymer have sufficient size and thus don't pass the membrane of the centrifuge filters.

\footnotetext{
${ }^{16}$ here called "PA" or "1xPA" for "polymer with ATTO dye".
} 

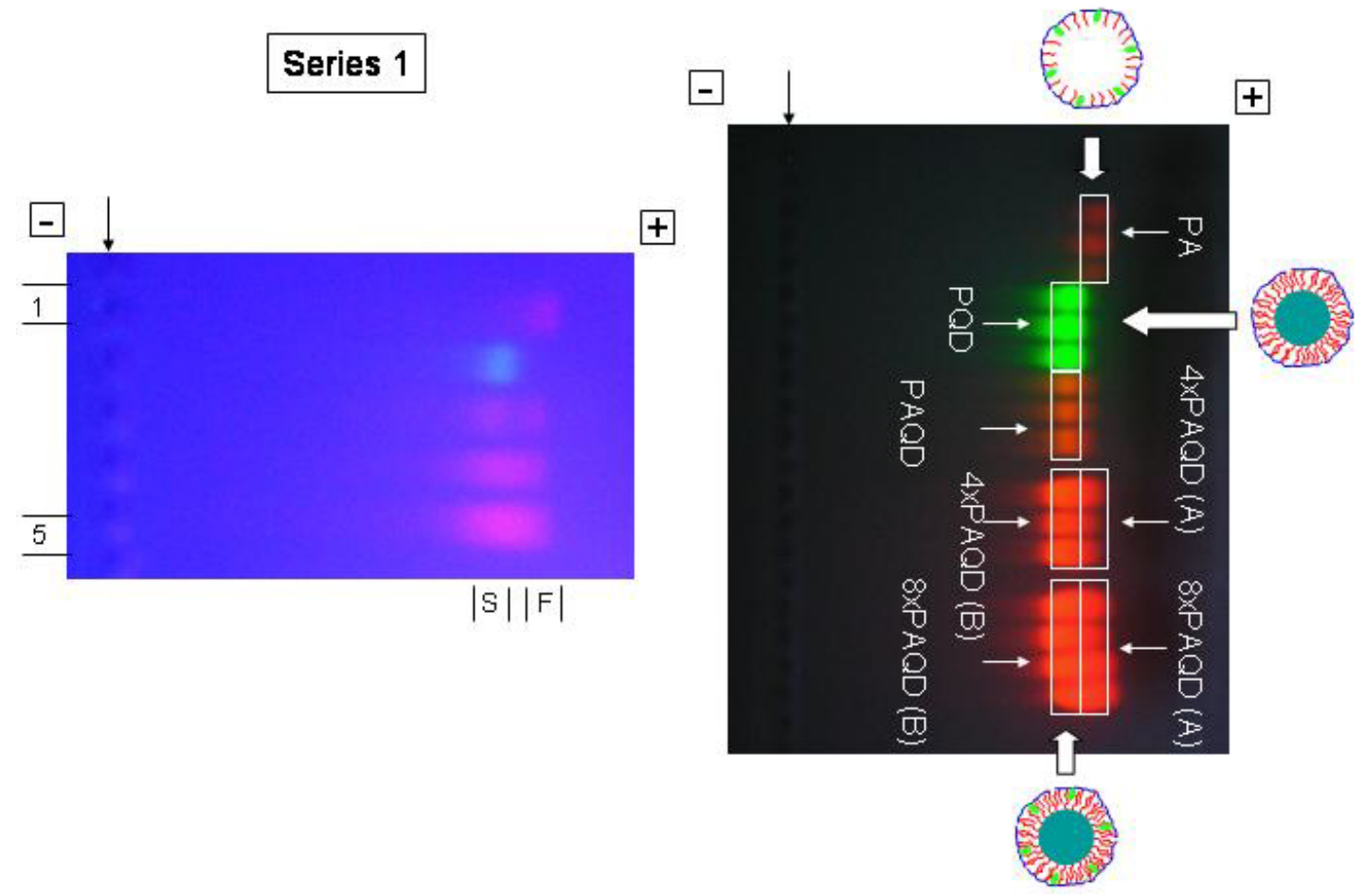

Fiqure SI-III.2: Images of 1-\% agarose gels on which different samples have been run. On the left gel each of the samples is loaded only in one lane, on the right gel the same sample had been loaded several time in adjacent lanes as to get a higher throughput. The samples in the lanes are, from top to bottom: PA (empty micelles of polymer with incorporated ATTO dye), PQD (polymer-coated quantum dots without dye in the polymer), PAQD (polymer-coated quantum dots with dye in the polymer), 4xPAQD (polymercoated quantum dots with dye in the polymer and $4 x$ the amount of polymer per quantum dot), $8 x P A Q D$ (polymer-coated quantum dots with dye in the polymer and $8 x$ the amount of polymer per quantum dot). Clearly slow ("S") and fast ("F") migrating bands can be identified in the left gel ${ }^{17}$. The polymer micelles $\left(" P A^{\prime \prime}\right)$ migrate faster than the polymer-coated quantum dots ("PQD"). In particular in the 3rd lane two bands can be seen ("PAQD"), one with slow, one with fast migration. The dotted lines on the right gel indicate the different fractions of sample that had been extracted from the gel. In the case of lanes 4 and 5 two fractions $(A, B)$ were extracted, which are attributed to the fast and the slow migrating bands.

Due to the fluorescence of the ATTO dye the polymer micelles appear with red fluorescence on the gel. In the second lanes of the gels a sample of polymer-coated quantum dots is shown, whereby no dye had been incorporated in this polymer $\left(\mathrm{F}_{0}\right.$ from Figure SI-II.1, see also Chapter II.2) ${ }^{18}$. Due to the fluorescence of the quantum dots this band appears green. In the following lanes polymer-coated quantum dot samples are shown, whereby ATTO-dye was incorporated in the polymer. Different amounts of polymer have been added to each sample. For example in the gel shown in Figure SIIII.3 in lane 5 the typical amount of polymer (see Chapter II.2) has been added. In lanes $3,4,6,7$ the amount of polymer that was added per particles was $0.3,0.5,2,4 \mathrm{x}$ the regular amount (the polymer-coated quantum dots correspond to $\mathrm{F}_{1}$ in Figure SI-II.1) ${ }^{19}$. The polymer micelle ("PA") migrates faster than the polymer-coated quantum dots ("PQD"). We attribute these mobilities to a slow ("S") and fast ("F") mobility. In Figures SI-III.2 and SI-III.4 it is shown, that when quantum dots are polymer-coated always two fractions result. Quantum dots embedded in a polymer-shell and empty

\footnotetext{
${ }^{17}$ More sample was loaded in each well of the right gel in order to get a higher throughput. This is the reason why the bands on the right are increasingly spread.

${ }^{18}$ here called "PQD" or "1XPQD" for "polymer around quantum dots"

${ }^{19}$ here called "XXPAQD" for "Xx the amount of polymer around the quantum dots, whereby ATTO dye was incorporated in the polymer.
} 
polymer micelles. If the polymer has ATTO dye embedded, then also the empty micelles can be observed by the fluorescence of the ATTO dye. From Figure SI-III.3 we can conclude that the typically used amount of polymer added per nanoparticles has been chosen well. In the case less polymer than usual is used to coat the quantum dots ("0.3xPAQD"), then the quantum dots transfer worse to aqueous solution. This can be seen in the very spread band, which indicates the existence of particles that are not well dispersed. On the other hand, for more polymer added than usual ("2xPAQD") most polymer does not wrap around the particles anymore, but just forms empty polymer micelles. This can be seen as the increased fraction of fast migrating particles, which we attribute to empty polymer micelles.
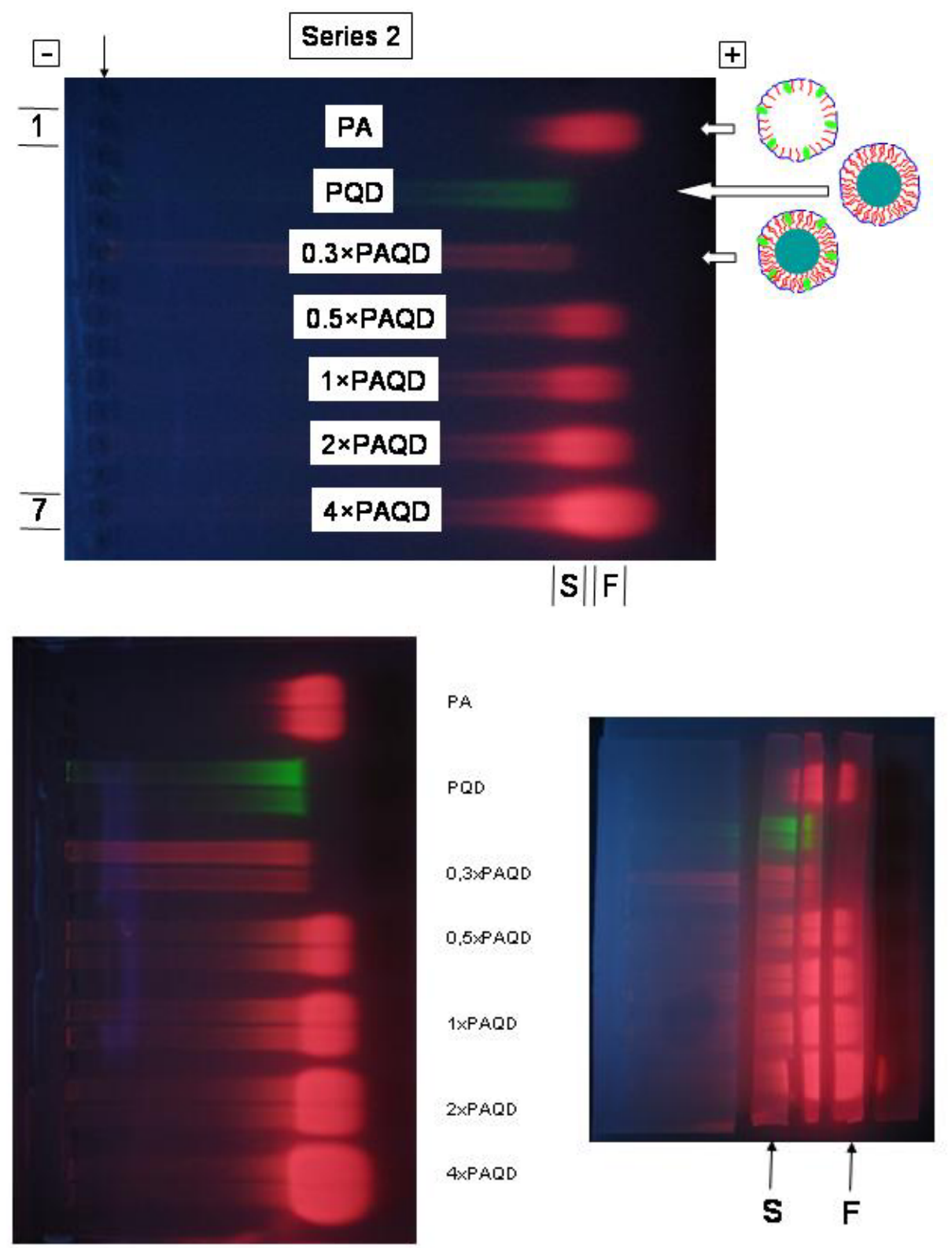

Fiqure SI-III.3: 1\% Agarose gel with different samples that had been run at $100 \mathrm{~V}$. Upper part: Test gel. From top to the bottom: polymer micelles with dye in the polymer, polymer-coated quantum dots, polymer-coated quantum dots with dye in the polymer with 0.3, 0.5, 1, 2, 4 times the amount of polymer per quantum dots which is regularly used. The arrow indicates the position where the samples had been loaded on the gel. Lower part: Gel with increased amount of loaded samples in order to extract and recover the particles after gel electrophoresis from the gel. The parts of the gel that have been cut in order to recover the particles are shown. 
As it can be seen in Figure SI-III.2 free polymer micelles with dye attached inside the polymer structure ("PA") ran faster than polymer coated quantum dots ("PQD"). This allowed us to separate the excess of empty polymer micelles (with dye in the polymer) from the polymer-coated quantum dots. For this the samples were first run on an agarose gel. Then the bands were cut out of the gel, whereby always as well the slow as the fast migrating fraction was cut. The two fraction that were recovered from the gel from samples "4xPAQD" and "8xPAQD" correspond to the empty polymer micelles (the upper fraction (A)), and the polymer-coated quantum dots (the lower fraction (B)). To recover sufficient amounts of sample always first one test gel with a small amount of sample was run to see the position of the bands. Then a preparative gel with the same sample on several parallel lanes and with more sample per well was run. In these gels it was not possible anymore to distinguish exactly between the slow and the fast band. The two fractions were cut according to the results of the first test gel with distinguishable bands. Extraction of the samples from the cut gel pieces was done by immersing the samples for 1 day in a big volume of SBB (sodium borate) buffer, $\mathrm{pH} 9$. During this time the particles diffused out of this gel. Afterwards, the gel pieces were removed and the solutions were filtrated through a $0.22 \mu \mathrm{m}$ filter in order to avoid small pieces of gel in the samples. As last step the samples were concentrated in a 100,000 MWCO Centricon centrifuge filter. Three batches of different samples were prepared ("series 1" see Figure SI-III.2, "series 2" see Figure SI-III.3, and "series 3" see Figure SI-III.4).
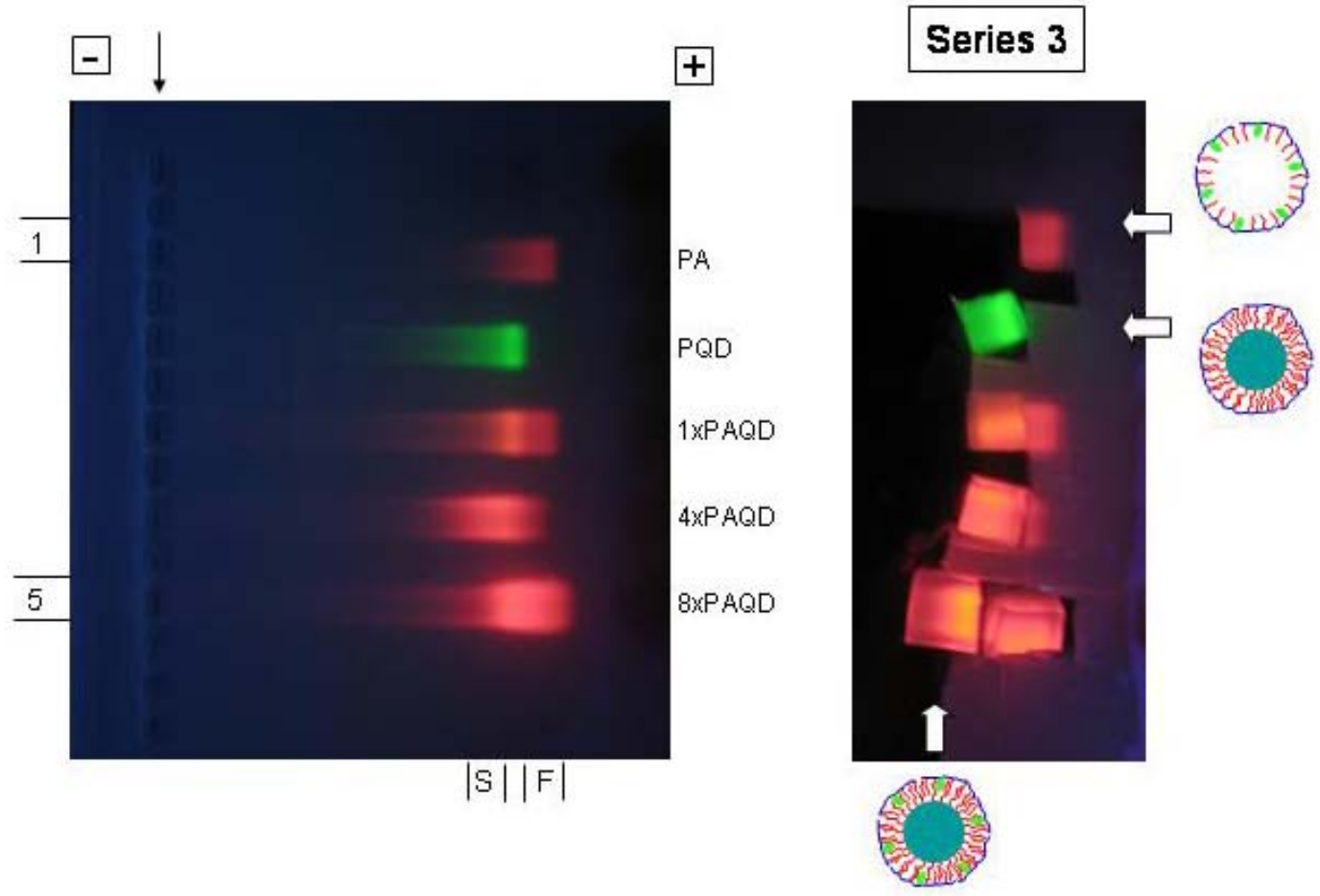

Fiqure SI-III.4: Similar experiment as described in Figure SI-III.2 for a different batch of particles ("series 3"). On the right side the pieces from the gel are shown (at higher illumination intensity) which had been used to extract the particles. In particular in lane 3 again the slow and the fast band can be seen. 


\section{III.3. Polymer-coated quantum dots with ATTO dye attached by post-modification}

Amino-modified ATTO dye was attached to polymer-coated quantum dots with EDC chemistry (see Chapter II.4). The effect of adding more and more ATTO dye per quantum dot can be seen in Figure SI-III.5. For high ATTO dye concentrations an additional fast band appears, which can be attributed to the empty polymer micelles. Polymer-coating of quantum dots always also results in some empty micelles as byproduct. However, as the polymer is not fluorescent they are not visible on the gel. Attachment of ATTO dye with EDC chemistry makes also the empty polymer micelles fluorescent and they can be identified by their red color on the gel. In order to investigate the nature of attachment amino-modified ATTO dye was also added to polymer-coated quantum dots without the addition of EDC. As can be seen in Figure SIIII.5 exactly the same results were obtained with and without the presence of EDC. This means, that amino-modified ATTO-dye sticks to polymer-coated quantum dots even without the presence of EDC. This can be understood taking into account electrostatic attraction and hydrophobic interaction. While amino-modified ATTO dye is positively charged (see Figure SI-III.1) the polymer-shell is negatively charged. In addition, the rather hydrophobic dye molecule could diffuse into the hydrophobic layer of the surfactant / polymer shell. Therefore, in principle no EDC is needed for attaching the ATTO dye. However, in all following experiments EDC was still added for the postconjugation of polymer-coated quantum dots with amino-modified ATTO-dye.
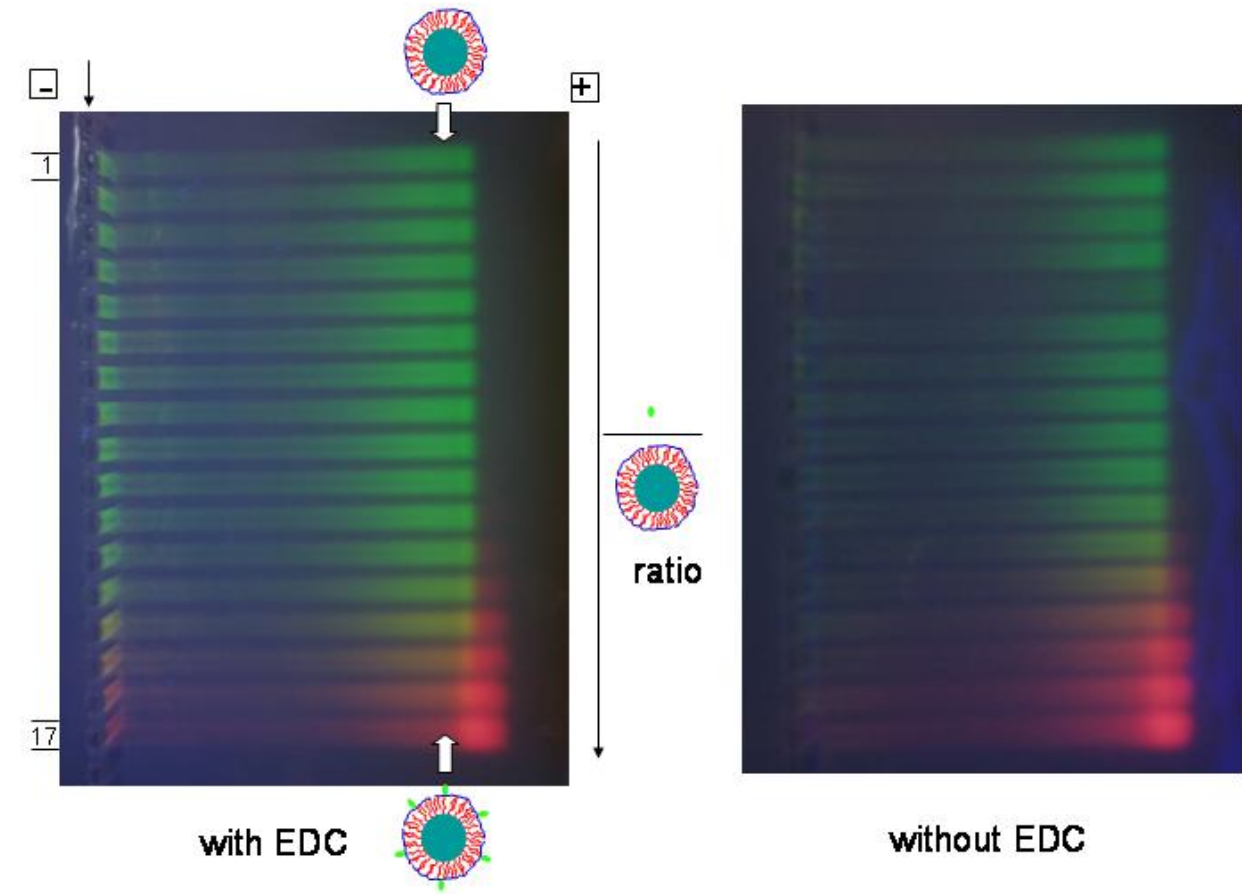

without EDC

Fiqure SI-III.5. Quantum dots were transferred to aqueous solution by coating them with an amphiphilic polymer without dye incorporated in the polymer ${ }^{20}$. A series was made in which different amounts of amino-modified ATTO dye was given to the polymer-coated quantum dots, ranging from $5 \times 10^{-3}$ to 320 ATTO molecules per quantum dot (from lane to lane the ATTO/quantum dot ratio is doubled). This was done under the presence of EDC (2000 EDC molecules per quantum dot) and without the presence of $E D C$. The samples were run on 1\% agarose gels and here the images of these gels under UV-excitation are shown. The amount of ATTO dye per quantum dot increases from lane 1 to lane 17. The addition of ATTO dye changes the color of the bands. However, there is no difference between the samples with and without EDC.

\footnotetext{
${ }^{20}$ This corresponds to sample "PQD" in Figure SI-III.3, SI-III.2, and SI-III.4.
} 
Two batches of samples ("series 1" and "series 2") were prepared. For each batch first a series was prepared, in which the amount of added amino-ATTO dye per quantum dot was gradually increased (Figure SI-III.6 and SI-III.8). From these series we selected the ATTO / quantum dot ratios for the samples which should be investigated.

After the ratios had been fixed new samples were prepared. For "series 1 " the ATTO / quantum dot ratios $0,10,40$, and 80 , and for "series 2" the ATTO / quantum dot ratios 0, 80, 160, and 320 were chosen. As shown in Figure SI-III-7 and SI-III-9 two fractions were recovered for each sample from the gels: the fast migrating upper and the slow migrating lower parts. Polymer coated quantum dots (PQD) move slower than empty micelles. Therefore, the samples were cut as indicated in the Figures in order to separate polymer coated particles from empty polymer micelles. The upper part of the bands correspond to the empty micelles with amino-modified ATTO dye attached onto their surface, and the lower fraction correspond to the polymer-coated quantum dots with amino-modified ATTO dye attached onto their surface. The samples were extracted from the gel pieces by soaking in SBB buffer of $\mathrm{pH}$, filtration through a $0.22 \mu \mathrm{m}$ filter, and concentration in a $100000 \mathrm{MWCO}$ Centricon centrifuge filter (see Chapter SI-III.3).

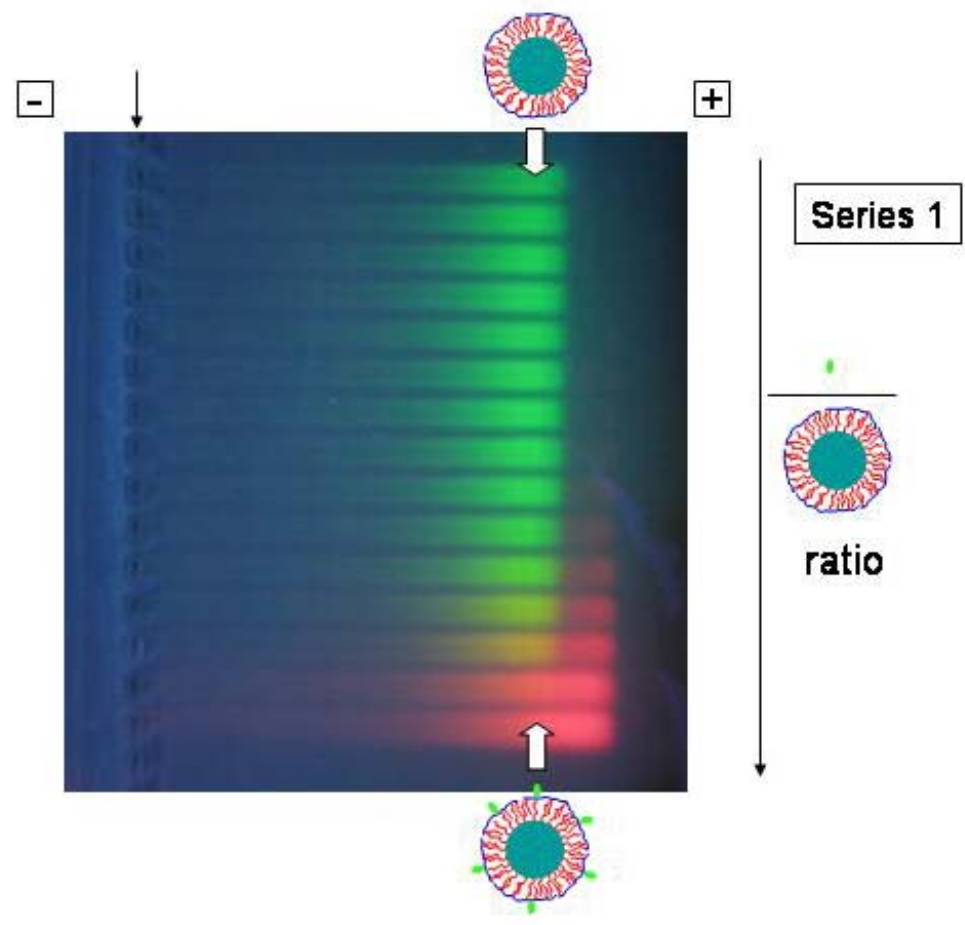

Fiqure SI-III.6: Quantum dots were transferred to aqueous solution by coating them with an amphiphilic polymer without dye incorporated in the polymer. A series was prepared in which different amounts of amino-modified ATTO dye was added to the polymer-coated quantum dots (from lane to lane the ATTO/quantum dot ratio is doubled). This was done under the presence of EDC (2000 EDC molecules per quantum dot). 

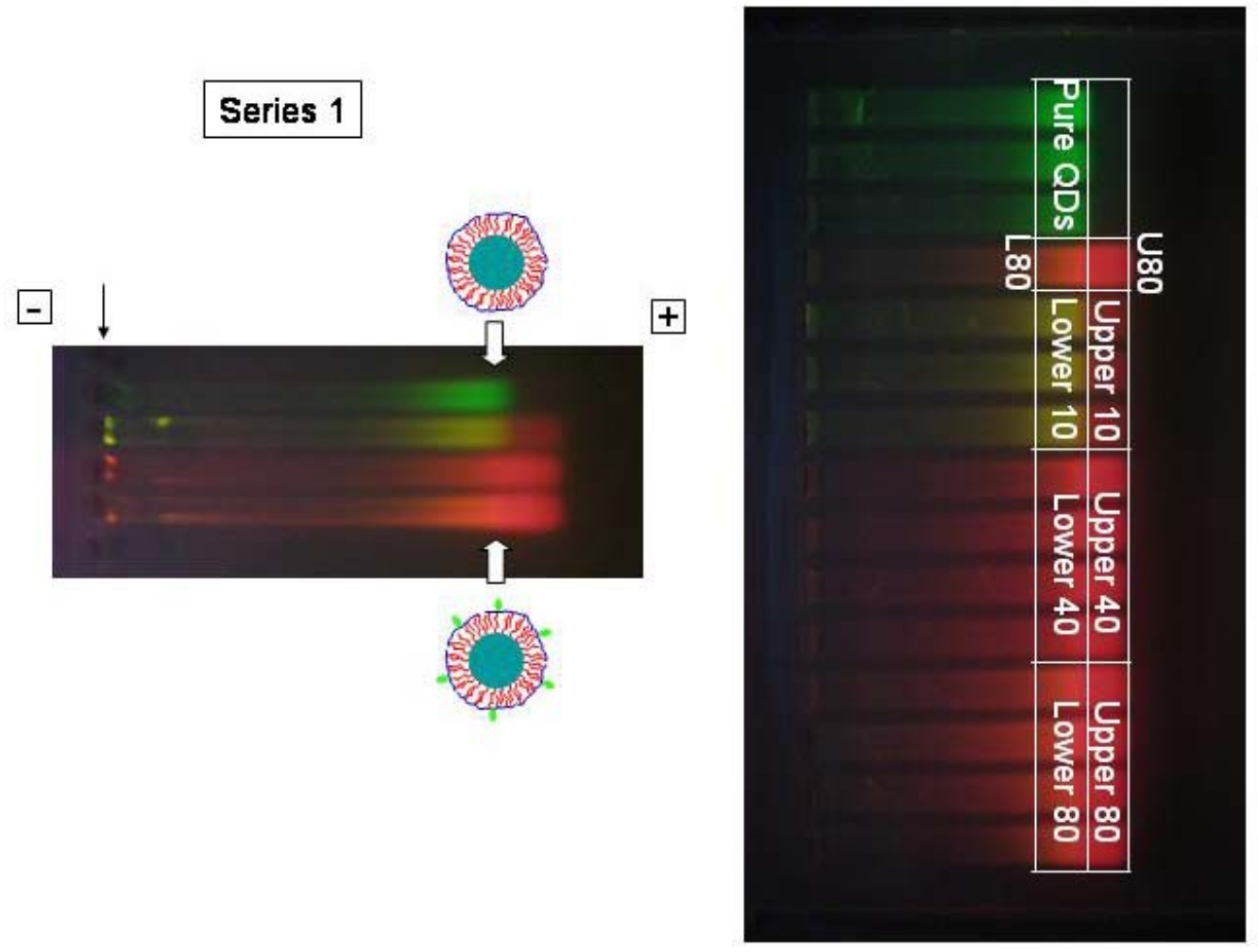

Figure SI-III.7: Amino-ATTO dye was attached to polymer-coated quantum dots with 0, 10, 40, and 80 added ATTO molecules per quantum dot under the presence of 2000 EDC molecules per quantum dot. The samples were then run on 1\% agarose gels. The left gel shows an overview, the preparative gel on the right shows several samples in parallel lines. From the right gel bands were cut out of the gel to recover the sample. The upper fast migrating as well as the lower slow migrating parts of the bands were extracted.

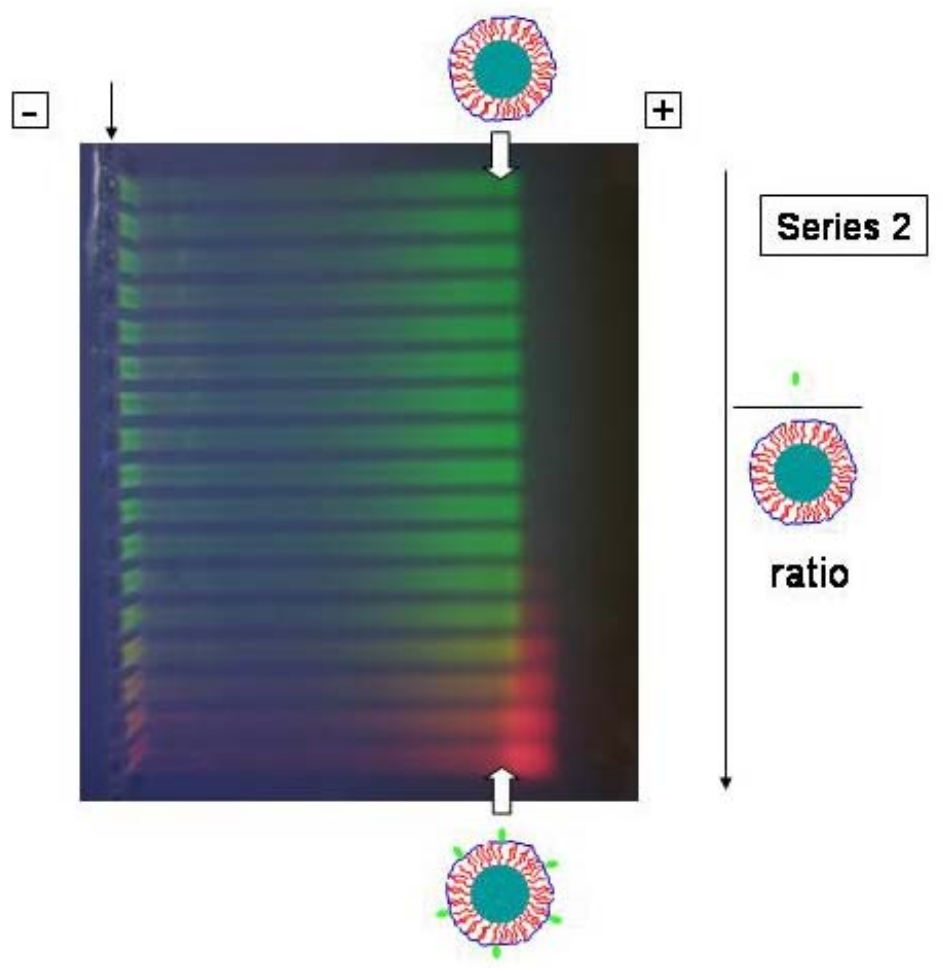

Fiqure SI-III.8: See the caption of Figure SI-III.6. 

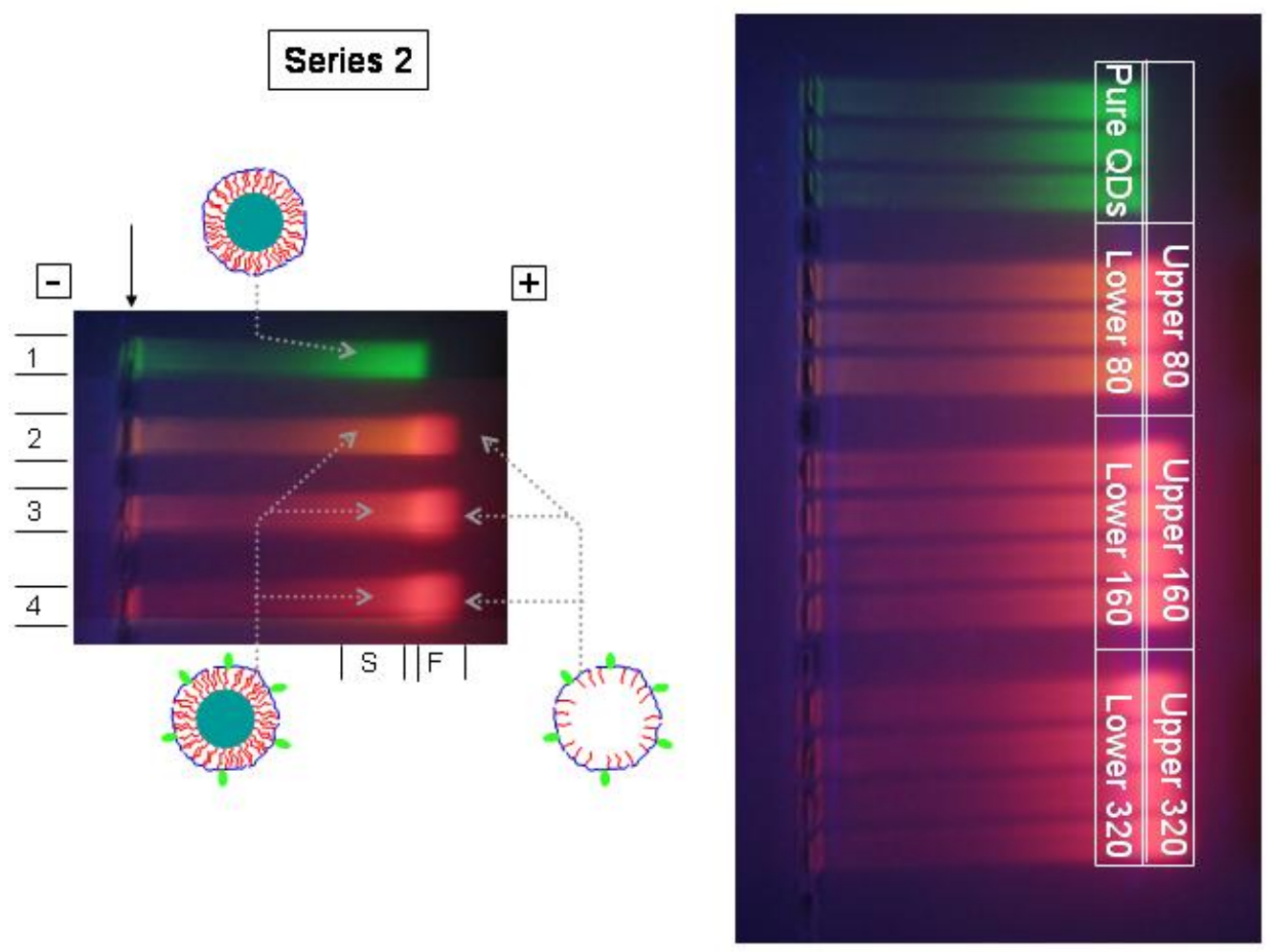

Figure SI-III.9: Amino-ATTO dye was attached to polymer-coated quantum dots with 0, 80, 160, and 320 added ATTO molecules per quantum dot under the presence of 2000 EDC molecules per quantum dot. The samples were then run on 1\% agarose gels. The left gel shows an overview, the right gels shows several samples in parallel lines. From the right gel bands were cut out of the gel to recover the sample. The upper fast migrating as well as the lower slow migrating parts of the bands were extracted.

\section{III.4. Polymer-coated quantum dots with ATTO dye attached to a shell of PEG}

NHS-modified ATTO dye was added to amino-PEG-coated quantum dots. The ATTO dye was attached via reaction of the NHS-ester with the free amines on the end of the PEGs that pointed towards solution. In Figure SI-III.10 the image of a gel is shown on which these conjugates had been run with different ATTO / quantum dot ratios. As well the bands of ATTO - quantum dot conjugates, as the bands of free NHS-ATTO dye (cfg. Figure SI-III.1) can be seen. A bigger batch of samples was then prepared with ATTO / quantum dot ratios of $0,2.7,10.9,21.8$, and 118 and the samples were run on a gel, see Figure SI-III.11. Two fractions (upper and lower part) were recovered from the bands as described in Chapter III.2. The upper part of the samples is attributed to the empty micelles with NHS-ATTO attached onto the PEG surface, and the lower fractions correspond to the quantum dots with NHS-ATTO bound onto the outer PEG shell. 


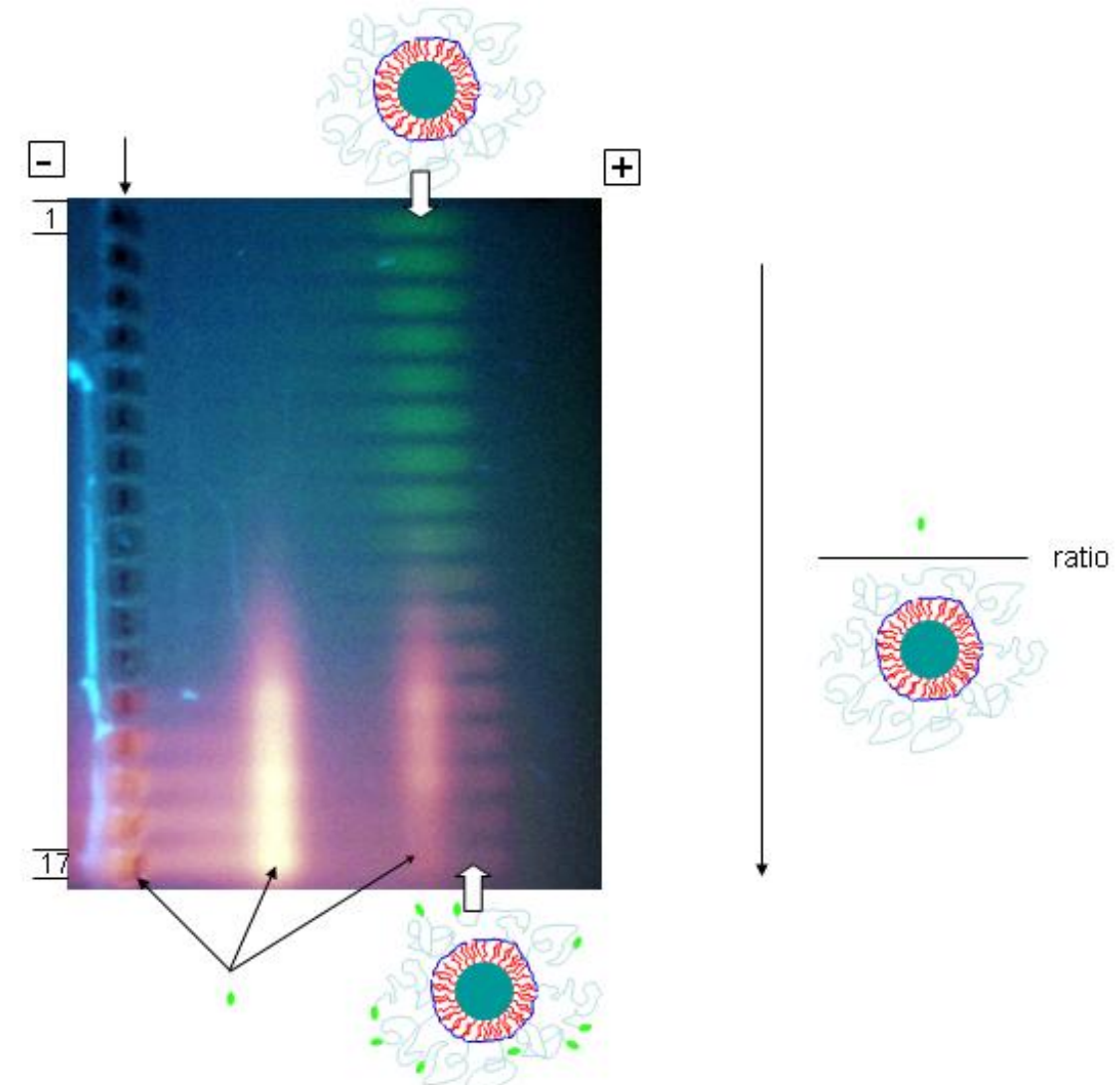

Fiqure SI-III.10: NHS-modified ATTO dye has been added to amino-PEG modified polymer-coated quantum dots. The samples were run after incubation on a 1\% agarose gel. From lanes 1 to 17 the ATTO I quantum dot ratio has been successively increased by a factor of two from lane to lane.
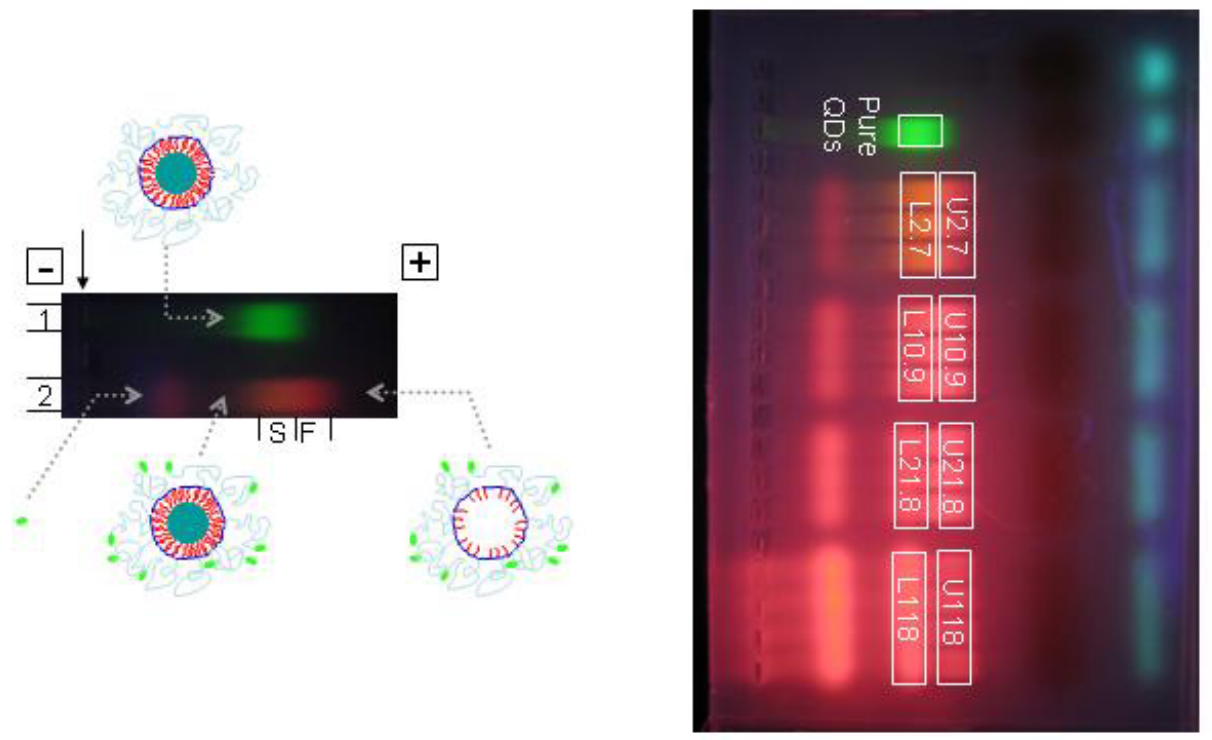

Fiqure SI-III.11: PEG-modified polymer-coated quantum dots were incubated with NHS-modified ATTO dye with the ATTO / quantum dot rations 2.7, 10.9, 21.8, and 118. These samples were run on 1\% agarose gel at $100 \mathrm{~V}$. The left gel shows the samples with PEG-modified quantum dots without and with the addition of ATTO dye (ATTO / quantum dot ratio = 2.7). The band of the free ATTO dye as well as a slow and fast band of particles can be observed. The fast band is attributed to empty micelles, and the slow band to polymer-coated quantum dots. On the right gel the samples that have been used for further measurements are shown. For this gel the lower and upper fractions of the bands were cut and the samples extracted and recovered. 


\section{Normalization procedure for the evaluation of fluorescence spectra}

All absorption measurements were done on an UV/Visible spectrophotometer (Ultrospec 3100 pro, Amersham Biosciences). Fluorescence spectra were recorded with a spectrofluorimeter (Fluoromax-3, Jobin Yvon Horiba) at two different excitation wavelengths, $450 \mathrm{~nm}$, in order to selectively excite the quantum dots, and $590 \mathrm{~nm}$ for the selective excitation of the ATTO-dye. The spectral characteristics of the samples were studied after running them through a S-400 size-exclusion column connected to a HPLC (1100 series, Agilent), and purification by gel electrophoresis.

For each series of sample (of approximately the same optical density) the following spectroscopic measurements were performed: recording of the absorption spectrum $(\mathrm{A}(\lambda))$, and two fluorescence spectra ${ }^{21}$, at $450-\mathrm{nm}\left(\mathrm{I}_{450}(\lambda)\right)$ and 590-nm excitation $\left(\mathrm{I}_{590}(\lambda)\right)$. In Figure SI-IV.1 the measurement protocol for the sample series shown in Figure SI-III.9 is depicted. In the first row of Figure SI-IV.1. the gel and the corresponding absorption spectra are shown. As in our experiments always many dye molecules were attached per quantum dot the absorption spectra were always dominated by the contribution of the dye ${ }^{22}$. Therefore we could not normalize the sample to solutions with the same quantum dot concentration, but we normalized to solutions with the same ATTO dye concentration. As most of the times only small amounts of samples were available, we could not always adjust all samples to exactly the same optical density of ATTO dye $\mathrm{e}^{23}$. Therefore we had to employ an additional step to normalize all the samples to the same concentration of ATTO dye.

At $590 \mathrm{~nm}$ the quantum dots virtually do not absorb, so that the absorption (and generated fluorescence) is dominated by the ATTO dye. We assume the ATTO fluorescence intensity to depend linearly on the concentration of ATTO dye. In this way, the concentration of ATTO dye in solution can be determined by measuring the

\footnotetext{
${ }^{21}$ Emission maxima of the quantum dots: $545 \mathrm{~nm}$, and of the ATTO dye: $622 \mathrm{~nm}$.

22 The first exciton peak in the absorption spectrum of the CdSe/ZnS quantum dots of $527 \mathrm{~nm}$ corresponds to molecular extinction coefficient of $77,800 \mathrm{M}^{-1} \mathrm{~cm}^{-1}$ at 527 (with neglecting the shift in the absorption peak due to the $\mathrm{ZnS}$ shell) (see Ref. \#4). For the recorded absorption spectra of the quantum dots the ratio of the absorption at $450 \mathrm{~nm}$ and $527 \mathrm{~nm}$, and the one of $590 \mathrm{~nm}$ and $527 \mathrm{~nm}$ is known, respectively (Figure SI-IV.1): $\mathrm{A}(450 \mathrm{~nm}) / \mathrm{A}(527 \mathrm{~nm})=2.0$, and $\mathrm{A}(590) / \mathrm{A}(527)=0.39$ and thus the molecular extinction coefficient of the quantum dots at $450 \mathrm{~nm}$ and $590 \mathrm{~nm}$ is $2.0 \times 77800 \mathrm{M}^{-1} \mathrm{~cm}^{-1}=$ $156000 \mathrm{M}^{-1} \mathrm{~cm}^{-1}$, and $0.39 \times 77800 \mathrm{M}^{-1} \mathrm{~cm}^{-1}=30300 \mathrm{M}^{-1} \mathrm{~cm}^{-1}$, respectively. The molar extinction coefficient of the dye at the absorption peak at $590 \mathrm{~nm}$ is according to the manufacturer $120000 \mathrm{M}^{-1} \mathrm{~cm}^{-1}$. From the recorded absorption spectrum of the dye (Figure SI-IV.1) the ratio of the absorption at $450 \mathrm{~nm}$ and $590 \mathrm{~nm}$ of dye is known: $\mathrm{A}(450) / \mathrm{A}(590)=0.18$ and thus the molecular extinction coefficient of the dye at $450 \mathrm{~nm}$ is $0.18 \times 120,000 \mathrm{M}^{-1} \mathrm{~cm}^{-1}=22,000 \mathrm{M}^{-1} \mathrm{~cm}^{-1}$. This means that the absorption of one quantum dot at $450 \mathrm{~nm}$ is roughly 7 times higher than the one of one dye molecule, whereas at $590 \mathrm{~nm}$ the absorption of one dye molecule is roughly 4 times higher that the one of one quantum dot. The noise level in the absorption measurement at $450 \mathrm{~nm}$ is about $15 \%$. As for the samples with quantum dots with attached ATTO dye no contribution of the quantum dots is visible in the absorption at $450 \mathrm{~nm}$, the contribution of the quantum dots can be at most the noise level of $15 \%$. As one quantum dot absorbs 7 times more than one dye molecule the maximum possible ratio of quantum dots to dye molecules is 0.15 / $7=0.021$. Or, in other words, at least $1 / 0.021=47$ dye molecules are attached per quantum dot.

${ }^{23}$ We always attempted to dilute all the samples within one series (e.g. all samples extracted from the gel shown in Figure SI-III.9) to the same optical density. However, sometimes one sample in one series could be extracted only with very low yield. Diluting all the samples of all series to this very low concentration would have involved a huge loss in intensity. Therefore, the samples of each series were diluted in a way that they had the optical density of the sample with the lowest concentration, but the optical densities between different series could vary.
} 
fluorescence intensity of $\mathrm{ATTO}^{24}$. As the quantum dots in first order do not absorb at 590-nm excitation, the ATTO emission upon 590-nm excitation does not depend whether they are present in the same solution or not. For this reason two fluorescence spectra were recorded from each sample (see second row of Figure SI-IV.1): one at 450 $\mathrm{nm}$ and one at 590-nm excitation. At 590-nm excitation to the first order only the ATTO dye absorbs and the emission maximum can be used to scale the recorded intensities in a way that they correspond to samples of the same ATTO-dye concentration. At $450 \mathrm{~nm}$ only the quantum dots absorb. Besides non-radiative recombination the absorbed energy can be either released upon photoluminescence emission of the quantum dots or upon energy transfer to attached ATTO dye and fluorescence emission of the ATTO dye. Therefore, the fluorescence emission upon 450-nm excitation is a measure for the FRET efficiency.

To normalize all samples to the same concentration of ATTO dye the emission spectra at $450 \mathrm{~nm}\left(\mathrm{I}_{450}(\lambda)\right)$ and $590 \mathrm{~nm}$ excitation $\left(\mathrm{I}_{590}(\lambda)\right)$ were divided by their respective maximum of the fluorescence intensity at $590 \mathrm{~nm}$ excitation $\mathrm{I}_{590}=\max \left(\left(\mathrm{I}_{590}(\lambda)\right)\right.$ :

$\mathrm{I}_{450}(\lambda) \Rightarrow \mathrm{I}_{450}(\lambda) / \mathrm{I}_{590} ; \quad \mathrm{I}_{590}(\lambda) \Rightarrow \mathrm{I}_{590}(\lambda) / \mathrm{I}_{590}$

The results of this normalization step are shown in the third line of Figure SI-IV.1. Now all the spectra correspond to solutions with the same concentration of ATTO dye. For this reason the spectra of the normalized ATTO emission upon excitation of the ATTO dye $\mathrm{I}_{590}(\lambda) / \mathrm{I}_{590}$ have all the same intensity ${ }^{25}$. The normalized emission $\mathrm{I}_{450}(\lambda) / \mathrm{I}_{590}$ upon $450 \mathrm{~nm}$ corresponds as well to the emission of solutions with the same amount of ATTO dye, regardless whether the ATTO dye is free in solution or attached to quantum dots. Within each series for which the optical densities have been adjusted to the same value this normalization step is sufficient to compare the fluorescence of all the different samples.

However, as the mean optical density of different series of samples could not be always adjusted to the same value, and since the $\mathrm{I}_{450} / \mathrm{I}_{590}$ ratio was found to slightly depend on the optical density a second normalization step had to be performed in order to compare the samples of different series with each other. In each series one sample with plain ATTO dye was included. As second normalization step now the emission intensities of each sample within one series are divided by the normalized intensity $\mathrm{I}_{450} / \mathrm{I}_{590}$ (ATTO) of the ATTO dye. In other words, the spectra of all samples within one series (for example the ones shown here in the third line) are all scaled with the same factor, so that after the second normalization (shown here in the forth line) the intensity of the free ATTO dye $\mathrm{I}(\mathrm{ATTO})=1$. The second normalization step does not change anything in the relation between the samples within one series (the diagrams in the 3rd and 4th line have exactly the same features), it only introduces an additional scaling factor, so that also the samples of different series (which were recorded at different optical densities) can be compared with each other. The resultant normalized intensity describes the

\footnotetext{
${ }^{24}$ Of course this "linearity" will be valid only in a very limited range. For this reason all the solutions of one series have to be diluted in a way that they have approximately the same optical density, i.e. they have similar absorption at the wavelength of the absorption maximum of the ATTO dye.

${ }^{25}$ This normalization procedure only works for samples in which the optical density is dominated by the ATTO dye. Naturally, for example a sample with only quantum dots but without ATTO dye, could not be normalized to any ATTO dye concentration.
} 
emission of samples normalized to the same concentration of ATTO dye at $450 \mathrm{~nm}$ excitation and normalized so that the emission of free ATTO dye is equal to 1.
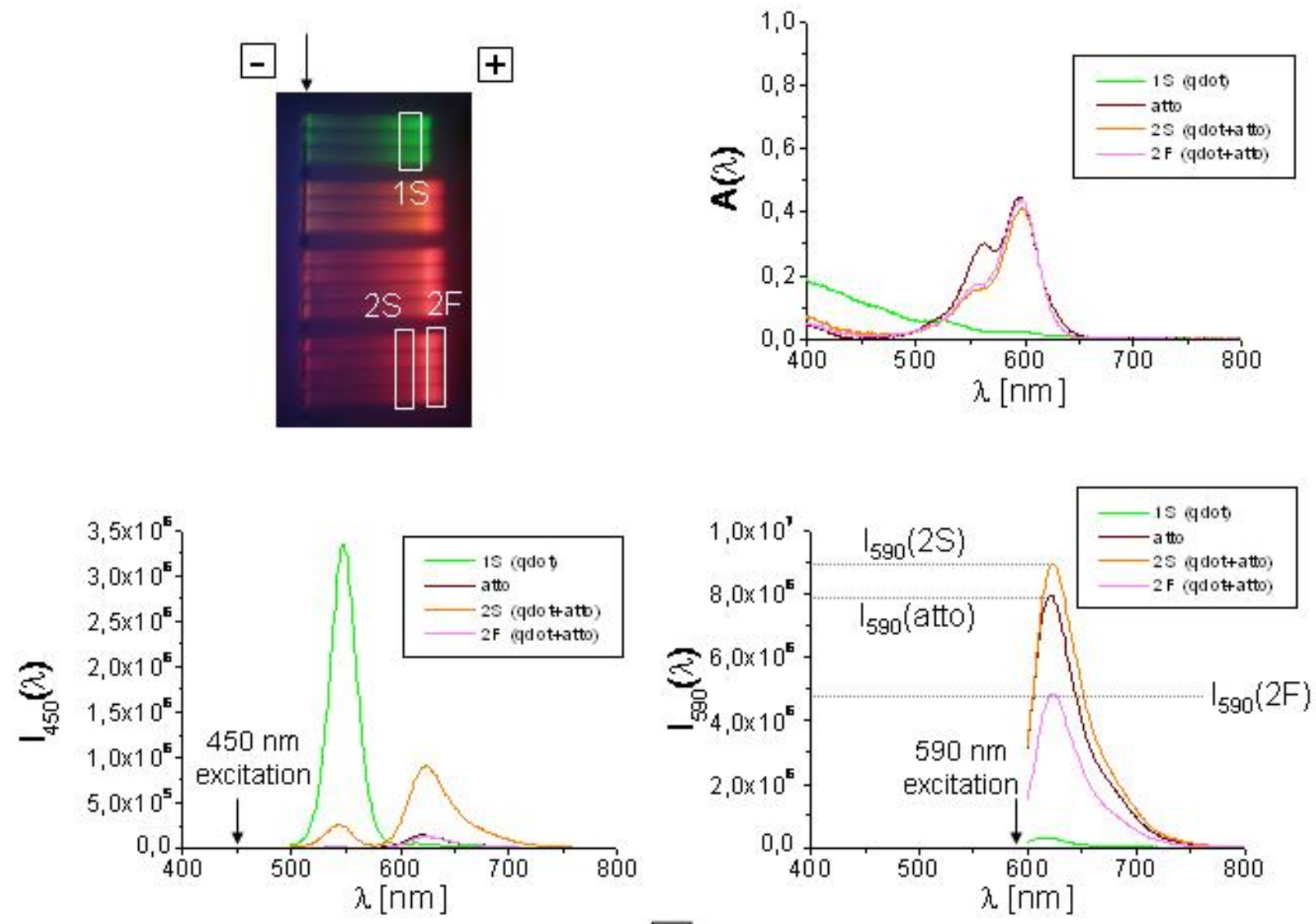

$\downarrow \begin{aligned} & \text { devide all intensities by } \\ & \mathrm{I}_{590} \text { of each sample }\end{aligned}$ 

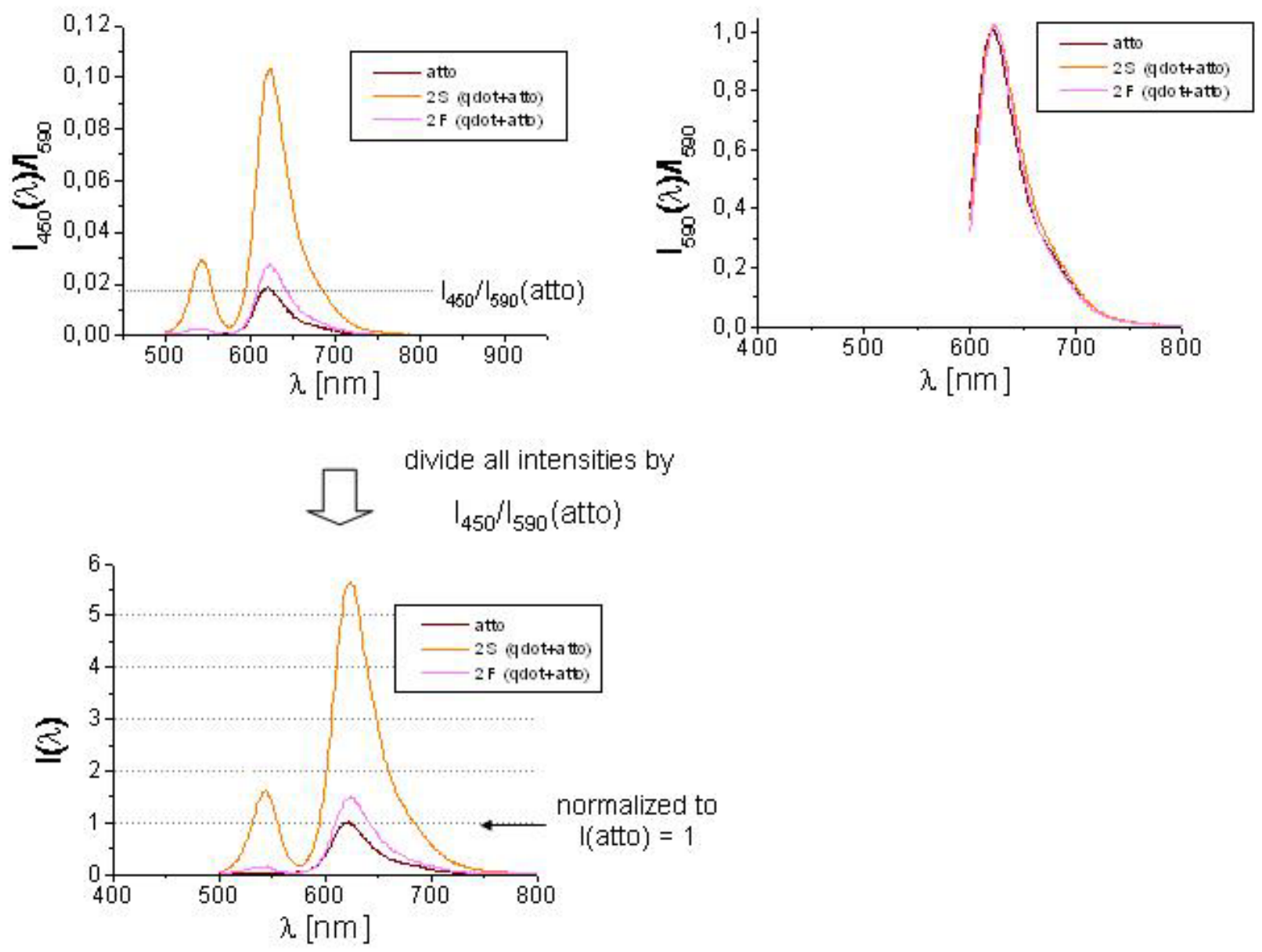

Fiqure SI-IV.1: Spectroscopic investigation of the samples. Of all samples that have been extracted from the gel (here: plain polymer coated quantum dots in the "slow" band of lane "1" (1S), polymer coated quantum dots with ATTO dye attached with EDC in the "slow" band of lane "2" (2S), empty polymer micelles with ATTO dye attached with EDC in the "fast" band of lane "2" (2F), all from the series shown in Figure SI-III.9; and as control free ATTO dye (atto)) absorption A( $\lambda$ ) spectra, and emission spectra at $450 \mathrm{~nm} I_{450}(\lambda)$ and at $590 \mathrm{~nm}$ excitation $I_{590}(\lambda)$ were recorded (first two lines). The emission intensities were normalized to correspond to solutions with the same concentration of ATTO dye by dividing them by the maximum intensity upon 590 excitation: $I_{450}(\lambda) \Rightarrow I_{450}(\lambda) / I_{590}, I_{590}(\lambda) \Rightarrow I_{590}(\lambda) / I_{590}$ (third line). In a last normalization step all the samples of one series were scaled with the same factor, so that the final normalized emission of free ATTO dye is 1 . 


\section{$\underline{\text { V. Evaluation of the fluorescence spectra }}$}

\section{V.1. Polymer-coated quantum dots with incorporated ATTO dye}

In Figures SI-V.1 - SI-V.3 the emission of free ATTO dye measured at $620 \mathrm{~nm}$ has been normalized to 1 (see Chapter IV). When ATTO-dye is embedded in the polymer that forms empty micelles the emission spectrum is not changed, i.e., a solution with polymer micelles with in total X embedded ATTO dye molecules has exactly the same fluorescence intensity as a solution with X free ATTO molecules (data not shown).

The fast band obtained from samples of quantum dots with ATTO dye embedded in the polymer shell appears always red on the gel. The normalized emission of this band is also 1 and its spectrum corresponds to the one of empty polymer micelles with incorporated ATTO dye. This is a very good indication that the fast bands of samples of polymer coated quantum dots correspond to unbound polymer that has formed empty micelles.

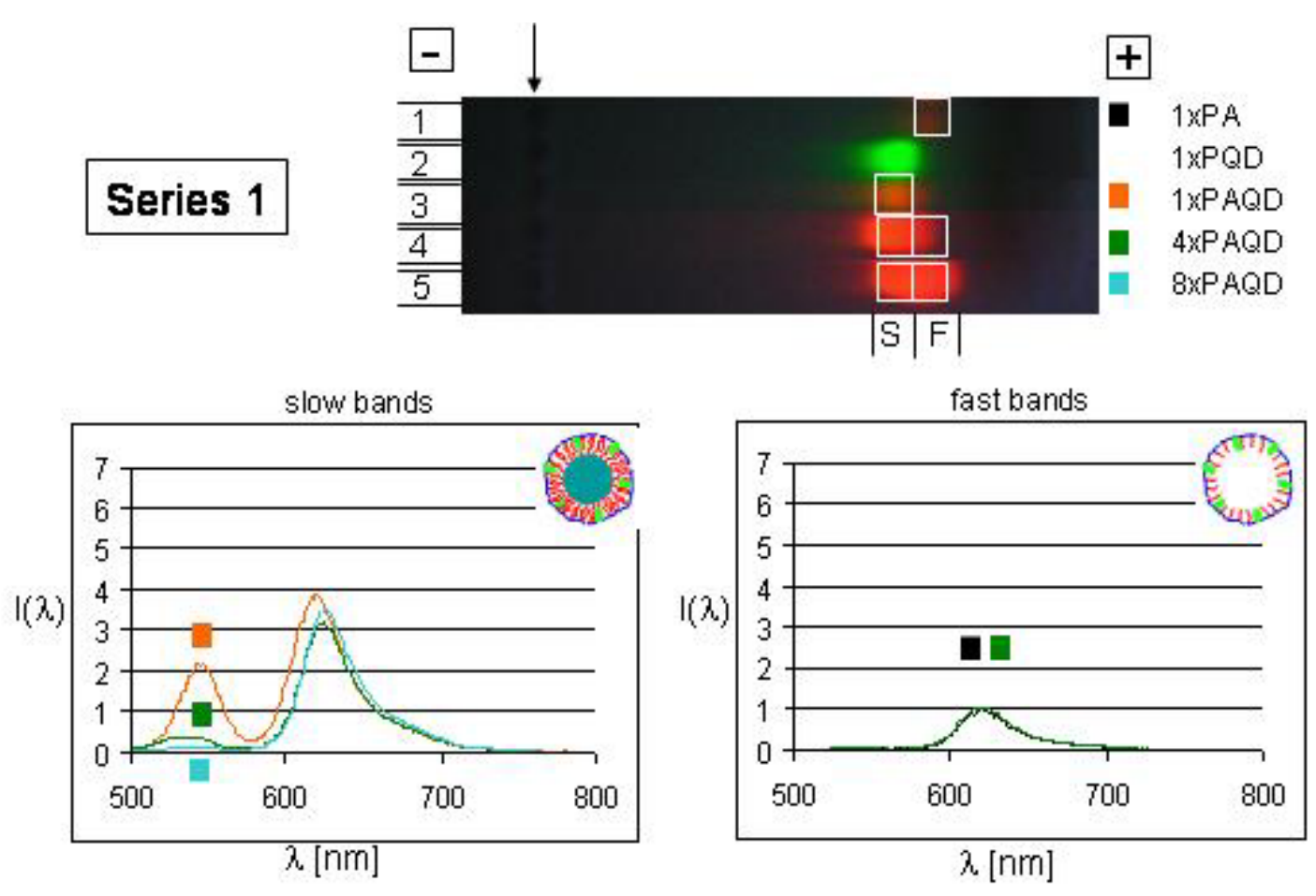

Fiqure SI-V.1. Normalized fluorescence emission spectra obtained for an excitation wavelength of 450 $n m$ for the following samples: PA, PAQD, 4xPAQD (A, B), and 8xPAQD (A, B). The slow ("S") bands correspond to quantum dots embedded in polymer micelles and the fast bands (" $F$ ") correspond to empty polymer micelles. The samples are described in Figure SI-III.2.

In contrast, the slow band of these samples displays in general two emission peaks. One at $545 \mathrm{~nm}$ (corresponding to the green emission of the quantum dots), and one at 620 $\mathrm{nm}$ (corresponding to the red emission of the ATTO dye). It is obvious from all three figures that the normalized emission intensity of the ATTO-modified quantum-dot samples is 3-6 times higher than the corresponding normalized emission of plain ATTO-modified empty micelles. This can be explained by the fact that quantum dots can absorb the light of the excitation source at $450 \mathrm{~nm}$ more efficiently than the ATTO dye due to a higher absorption cross-section. Part of this energy is then transferred to the ATTO dye in a FRET process from where it is emitted under the emission of 
fluorescence light. The results illustrate as well that the quantum dots have been saturated with ATTO dye. Even if more ATTO dye is attached per quantum dot (see the series 1xPAQD, 2xPAQD, 4xPAQD, 8xPQQD where for each subsequent sample the number of ATTO molecules per quantum dot is doubled) the normalized emission intensity does not increase further. In other words, the ATTO dye molecules can't receive more energy from the quantum dots. On the other hand, the remaining quantum dot emission decreases upon the attachment of more ATTO molecules (see Figure SIV.1). In case of sufficient attachment of ATTO there is no more radiative emission of the quantum dots at $545 \mathrm{~nm}$, but all energy is either transferred to the ATTO dye molecules or lost in other non-radiative processes (quenching).
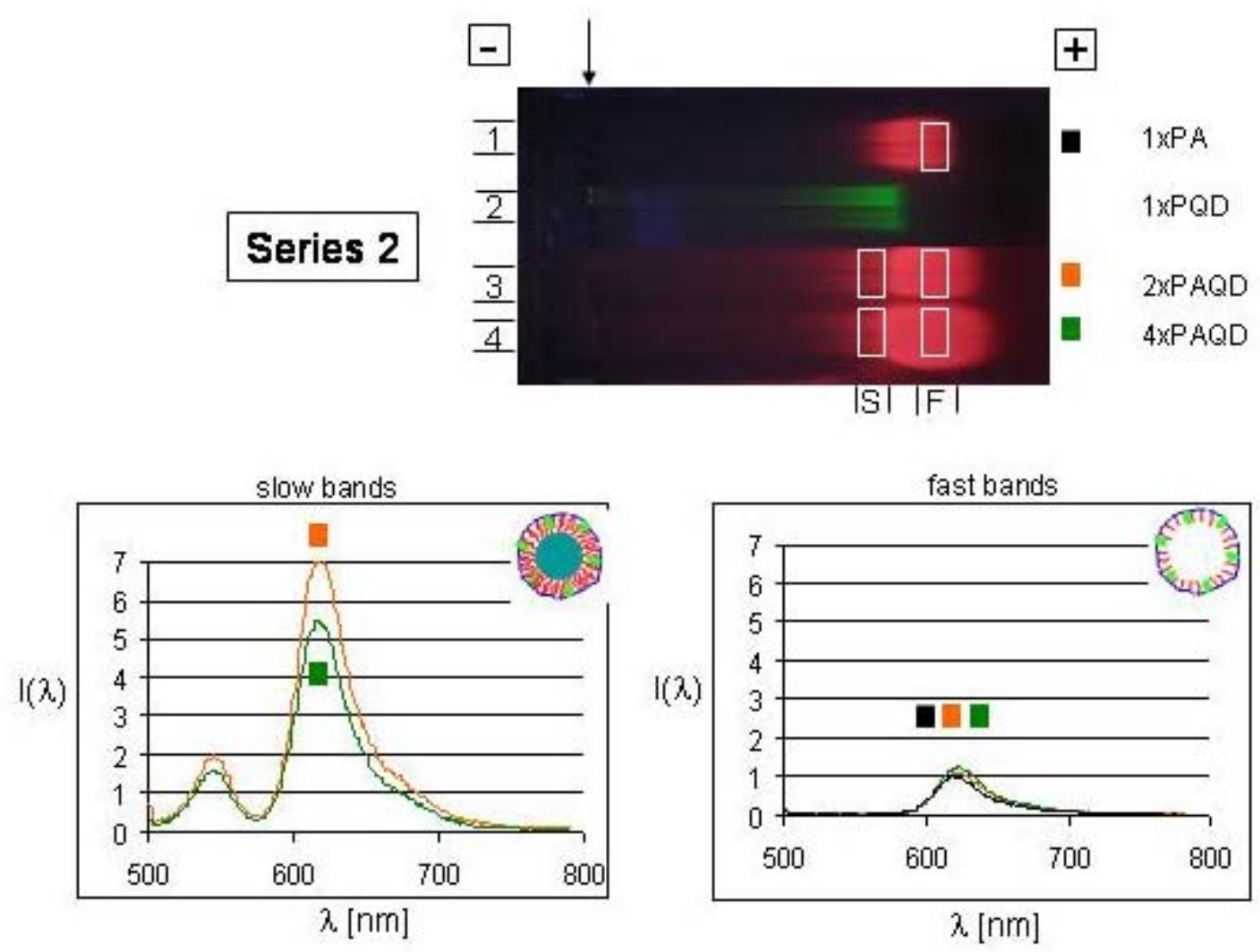

Fiqure SI-V.2. Normalized fluorescence emission spectra obtained for an excitation wavelength of 450 nm for the following samples: $P A, 2 x P A Q D$, and $4 x P A Q D$. The slow ("S") bands correspond to quantum dots embedded in polymer micelles and the fast bands $\left(" F^{\prime \prime}\right)$ correspond to empty polymer micelles. The samples are described in Figure SI-III.3. 

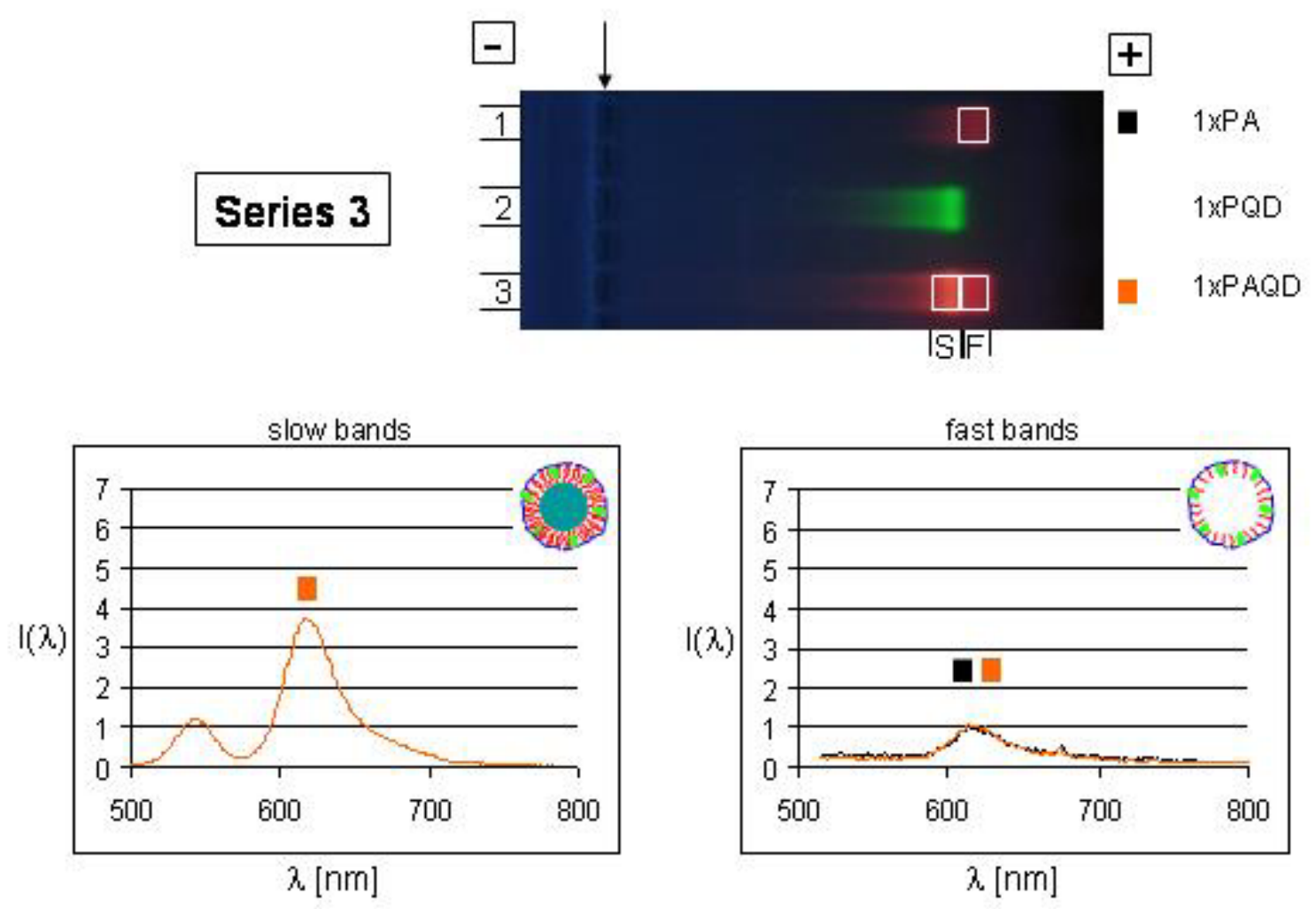

Fiqure SI-V.3. Normalized fluorescence emission spectra obtained for an excitation wavelength of 450 nm for the following samples: $P A$, and $P A Q D$. The slow ("S") bands correspond to quantum dots embedded in polymer micelles and the fast bands ("F") correspond to empty polymer micelles. The samples are described in Figure SI-III.4.

\section{V.2. Polymer-coated quantum dots with ATTO dye attached in post modification}

ATTO-dye has been attached with EDC chemistry to polymer-coated quantum dots. As described in the previous chapter, a fraction of empty polymer micelles inevitably coexists with the polymer-coated quantum dots. As both, quantum dots and empty micelles are labeled with ATTO dye, 2 bands appear on the gel.

It can be seen in Figures SI-V.4 and SI-V.5 that the normalized emission at $620 \mathrm{~nm}$ of the upper fractions, U10, U40 and U80 is lower than the emission of the lower fractions L10, L40 and L80. The normalized fluorescence of the upper band is the same as the one of free ATTO dye, see Figure SI-V.5. This can be explained if we consider that the upper part of the gel corresponds to empty polymer micelles with dye attached onto their shell. Therefore, in the absence of donor in empty micelles, the emission of the ATTO dye upon 450-nm excitation is the same as the one of free ATTO dye. Again, this corroborates our interpretation that the upper band corresponds to empty micelles.

The lower fractions contain quantum dots with ATTO dye attached onto the outer shell of the polymer. As described in the previous chapter, 450-nm excitation results in FRET, which results in a higher normalized emission ("sensitized acceptor fluorescence") at $620 \mathrm{~nm}$, compared to the same concentration of free ATTO dye. 

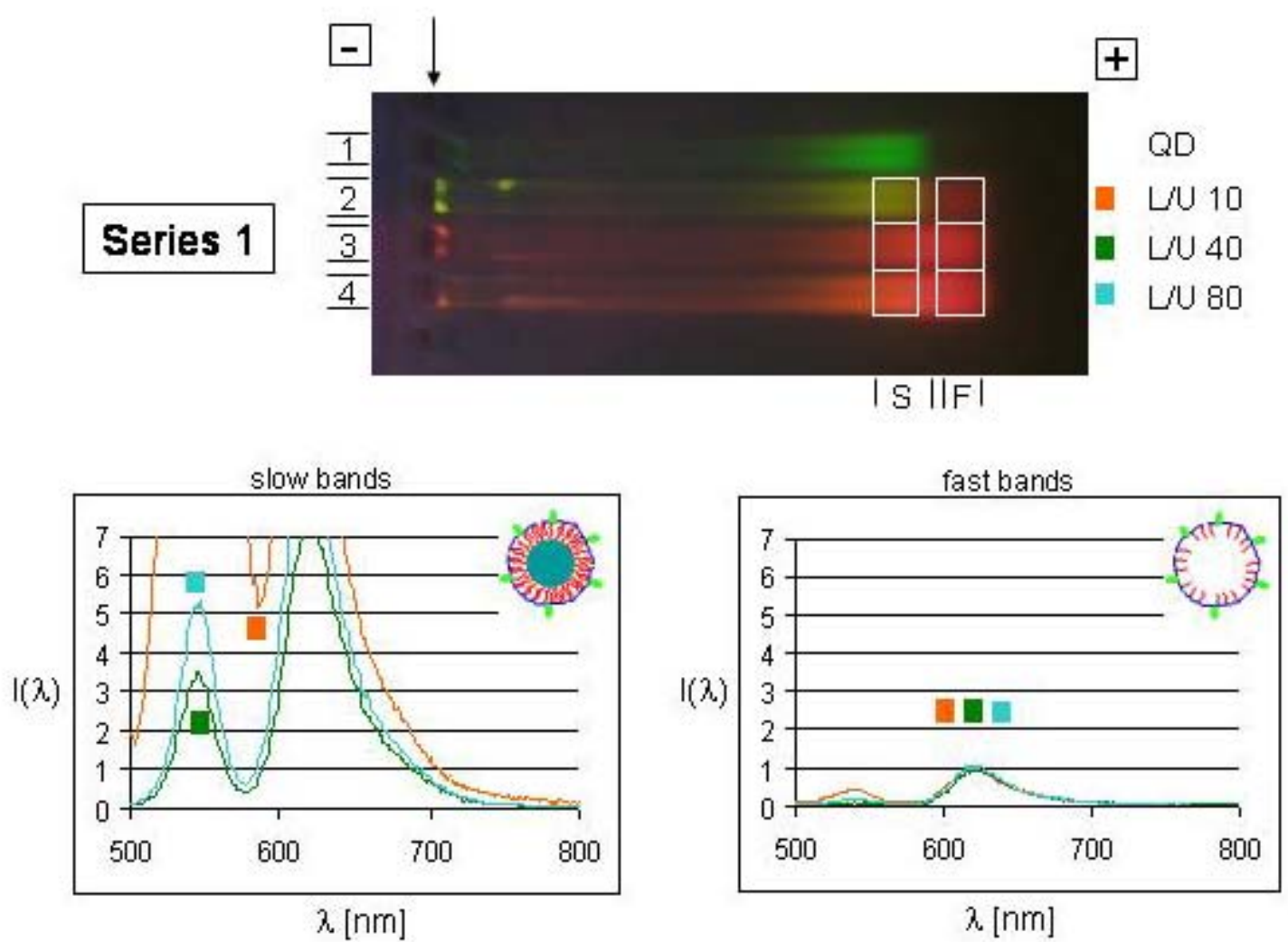

Fiqure SI-V.4. Normalized fluorescence emission spectra obtained at an excitation wavelength of 450 nm. The different samples are described in Figure SI-III.7. Fast ("F") upper ("U"), as well as slow ("S") lower ("L") bands of the samples were measured.
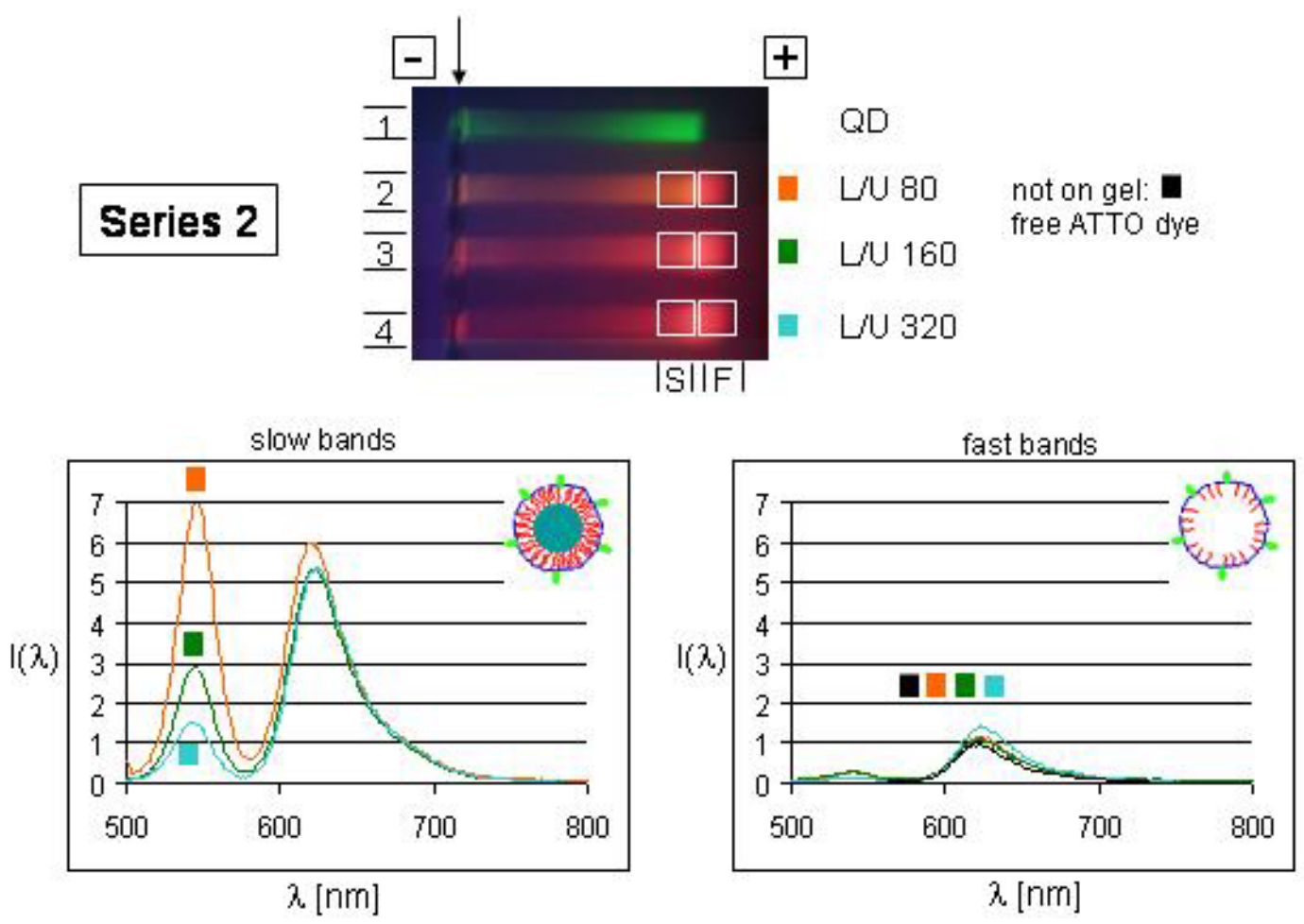

Figure SI-V.5. Normalized fluorescence emission spectra obtained at an excitation wavelength of 450 $n m$. The different samples are described in Figure SI-III.9. As well the fast ("F") upper ("U"), as the slow ("S") lower (" $L ")$ bands of the samples were measured. 
In Figure SI-V.4 sample "L10" one problem of the here applied normalization is apparent. In the case that a sample contains only few ATTO dye molecules compared to the amount of quantum dots, the normalization can't be applied anymore, as it is based on the ATTO absorption at $590 \mathrm{~nm}$. In this case, division through small numbers yields apparent normalized fluorescence intensities with aberrant high values. Both in Figure SI-V.4 and Figure SI-V.5 the quantum dots are saturated with ATTO dye. More ATTO dye per quantum dot $(\mathrm{L} 10 \rightarrow \mathrm{L} 40 \rightarrow \mathrm{L} 80 \rightarrow \mathrm{L} 160 \rightarrow 320)$ does not lead to a higher normalized emission at $620 \mathrm{~nm}$, but leads to a lower normalized emission at $545 \mathrm{~nm}$ upon excitation at $450 \mathrm{~nm}$.

\section{V.3. Polymer-coated quantum dots with ATTO dye attached to a shell of PEG}

As a control, we equally prepared donor/acceptor conjugates in which the FRET efficiency should be decreased by adding an additional spacer between the donor and acceptor. To this end, the QD545 particles were first coated with a polymer shell devoid of dye, the polymer surface was saturated with a monolayer of amino-modified PEG, and finally NHS-modified ATTO590 dye was added to the PEG by reaction of the NHS-ester with the amino groups of the PEG. Due to the PEG, these conjugates have a very high colloidal stability, close to that of the water-soluble quantum dots distributed by commercial vendors. However, the PEG shell significantly increases the thickness of the shell around the NC. We again took the difference between the hydrodynamic radius of the PEG- and polymer-coated particles and the radius of the inorganic $\mathrm{CdSe} / \mathrm{ZnS}$ quantum dots as an upper bound for the distance between the dye and the NC surface. Measurements with different techniques suggested that the maximum distance between dye and quantum dot surface is of the order of 9-10 nm for PEG of $20 \mathrm{kDa}$ molecular weight ${ }^{5}$. As the PEG on the quantum dot surface is randomly coiled and the ATTO dye is attached to the free end of the PEG there will be again a distribution of donor/acceptor distances. However, data derived on similar systems indicate that this distribution might be narrower than one intuitively could expect ${ }^{6}$. Nevertheless, the PEG significantly increased the donor/acceptor distance and less efficient FRET is observed was observed (Figure SI-V.6). Although we still observed quenching of donor fluorescence, the normalized sensitized ATTO dye fluorescence was lower than in the configurations where the dye was bound to the polymer shell, either directly embedded in the polymer shell or to its outside. Time-resolved fluorescence measurements corroborated these observations (Figure SI-VI.4). The observed distance dependence lends further support to the assumed FRET mechanism. However, for two reasons we think that the data obtained with PEG-coated particles are too preliminary to be included in the main paper. First, in the gels it was hard to attribute the smeared (?) bands to certain species, in particular since already the free NHS-modified ATTO-dye showed three bands upon gel electrophoresis. Second, as we could not determine precisely the donor/acceptor ratio, the samples involving PEG-coated quantum dots cannot be directly compared with the other conjugates. Indeed, the number of ATTOdye molecules per quantum dot might be significantly less than in the other cases in which the ATTO dye has been directly incorporated in the polymer shell or attached to the polymer shell with EDC. 
The results for ATTO dye attached to a PEG shell around polymer-coated quantum dots are shown in Figure SI-V.6. The normalized emission of sample "U2" corresponds to the normalized emission of free ATTO dye.

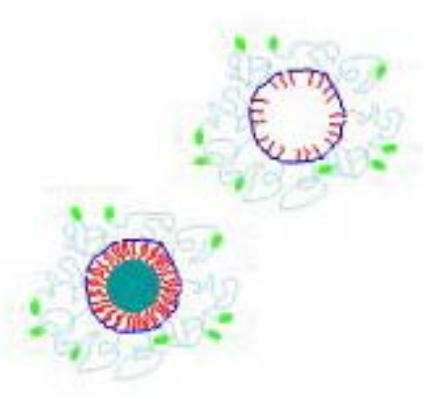

slow bands

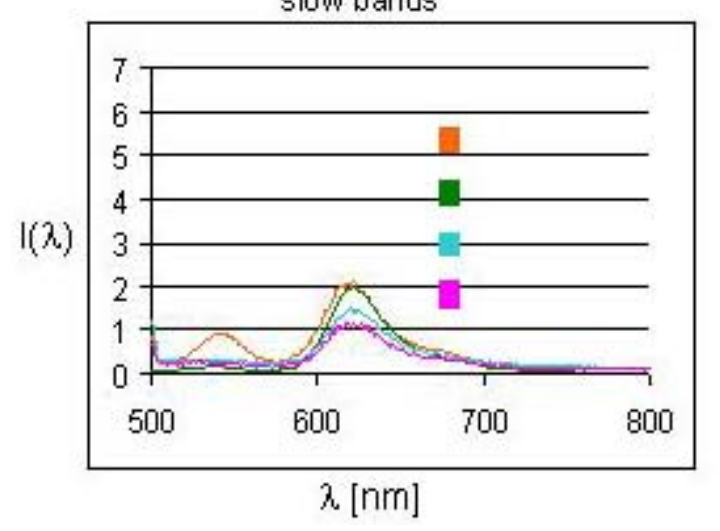

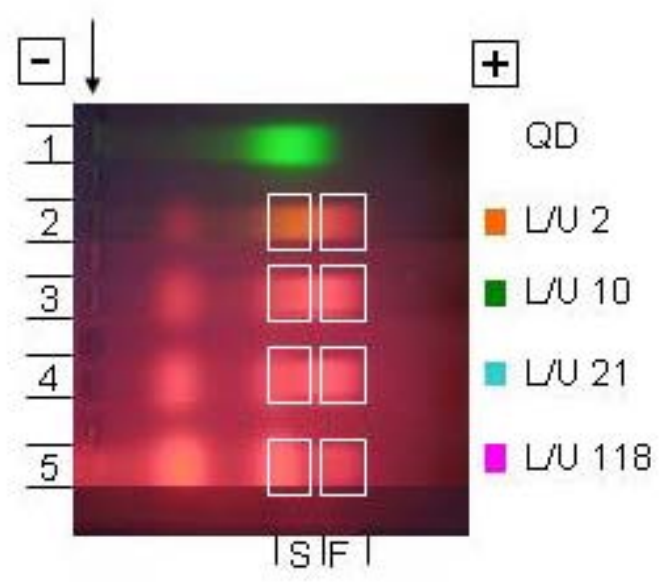

festibands

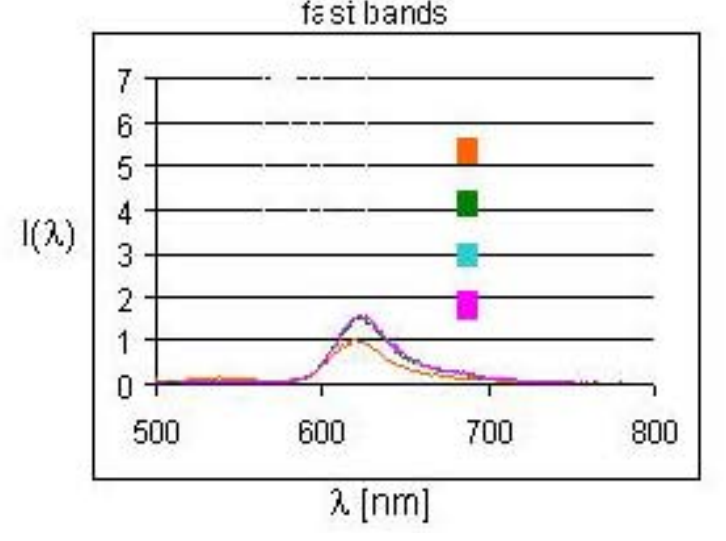

Fiqure SI-V.6. Normalized fluorescence emission spectra obtained at an excitation wavelength of 450 $n m$. The different samples are described in Figure SI-III.11. As well the fast ("F") upper ("U"), as the slow ("S") lower ("L") bands of the samples were measured. 


\section{Time resolved measurements}

\section{VI.1. Experimental set-up}

We also characterized the FRET efficiencies of the different donor/acceptor assemblies by recording the photoluminscence decays, using time-correlated single photon counting (TCSPC) technique. We used a Ti-Sapph subpicosecond pulsed laser (Tsunami, Spectra Physics, Mountain View, CA), running at 900-nm, in conjunction with a second harmonic generation frequency doubler and pulse picker to provide 450$\mathrm{nm}$ pulses at $4 \mathrm{MHz}$. The fluorescence emission of the quantum dots was selected by a monochromator (SpectraPro 150, ARC, $545 \mathrm{~nm}, \Delta \lambda=15 \mathrm{~nm}$ ) and detected by a photomultiplier (HamamatsuR3809U-50). A TCSPC board (SPC-430, Becker-Hick1 $\mathrm{GmbH}$, Berlin, Germany) was used for the acquisition of both excitation light pulse and photoluminescence decays. The time scaling was $37.4 \mathrm{ps} / \mathrm{channel}$ and 4096 channels were used. The function of the instrumental response of the laser pulse (100ps FWHM) was alternatively recorded with fluorescence by detecting the light scattered in water. If necessary, an optical attenuator was introduced in the excitation path to bring the detected intensity below 30,000 counts/s to avoid pile-up of photons. Acquisition cycles were maintained as to reach about 150,000 counts in the peak channel (corresponding to about half- to one-hour acquisition per sample). Experiments were done at $20^{\circ} \mathrm{C}$, using $3 \times 3-m m$ suprasil cuvettes, with an arbitrary dilution of 1/35 of the initial samples used for the measurement shown in Chapter V.

Analysis of the fluorescence decay was carried out using the maximum entropy method 7,8. After excitation by short pulse of the laser, the excited state of the quantum dot donors typically decays with a multi-exponential time course. Two short lifetimes in the range of $0.2-0.3 \mathrm{~ns}$ and $0.8-0.9 \mathrm{~ns}$ and a third long lifetime component ranging from 13 to $21 \mathrm{~ns}$. In this way, three main constants for relaxation of the excited quantum dots samples with / without attached ATTO dye were obtained for each sample.

The results for the different series of samples that had been introduced in Chapter III are presented in the following.

\section{VI.2. Polymer-coated quantum dots with incorporated ATTO dye}

In the absence of ATTO acceptor dye, the average fluorescence lifetime of quantum dots with polymer shell is around $10 \mathrm{~ns}$. In presence of ATTO acceptor, the excited state lifetime of the quantum dot donor fluorescence is shortened by FRET that offers an alternative route for de-excitation and relaxation to the ground state. The reduction of the photoluminescence lifetime depends on the number of attached acceptor molecules. This observation is understood when considering FRET as a bi-molecular process between a donor and acceptor each having a given orientation and donor/acceptor distance. Multiple acceptors provide, on average, shorter distance and more advantageous orientations for efficient non-radiative energy transfer. Figure SI-VI.1 displays the fluorescence decay curves of quantum dots at various ATTO/quantum dots ratios. The derived decay times are listed in Table SI-IV.1. The way how the decay times are extracted from the data is described in detail elsewhere ${ }^{7,8}$. 

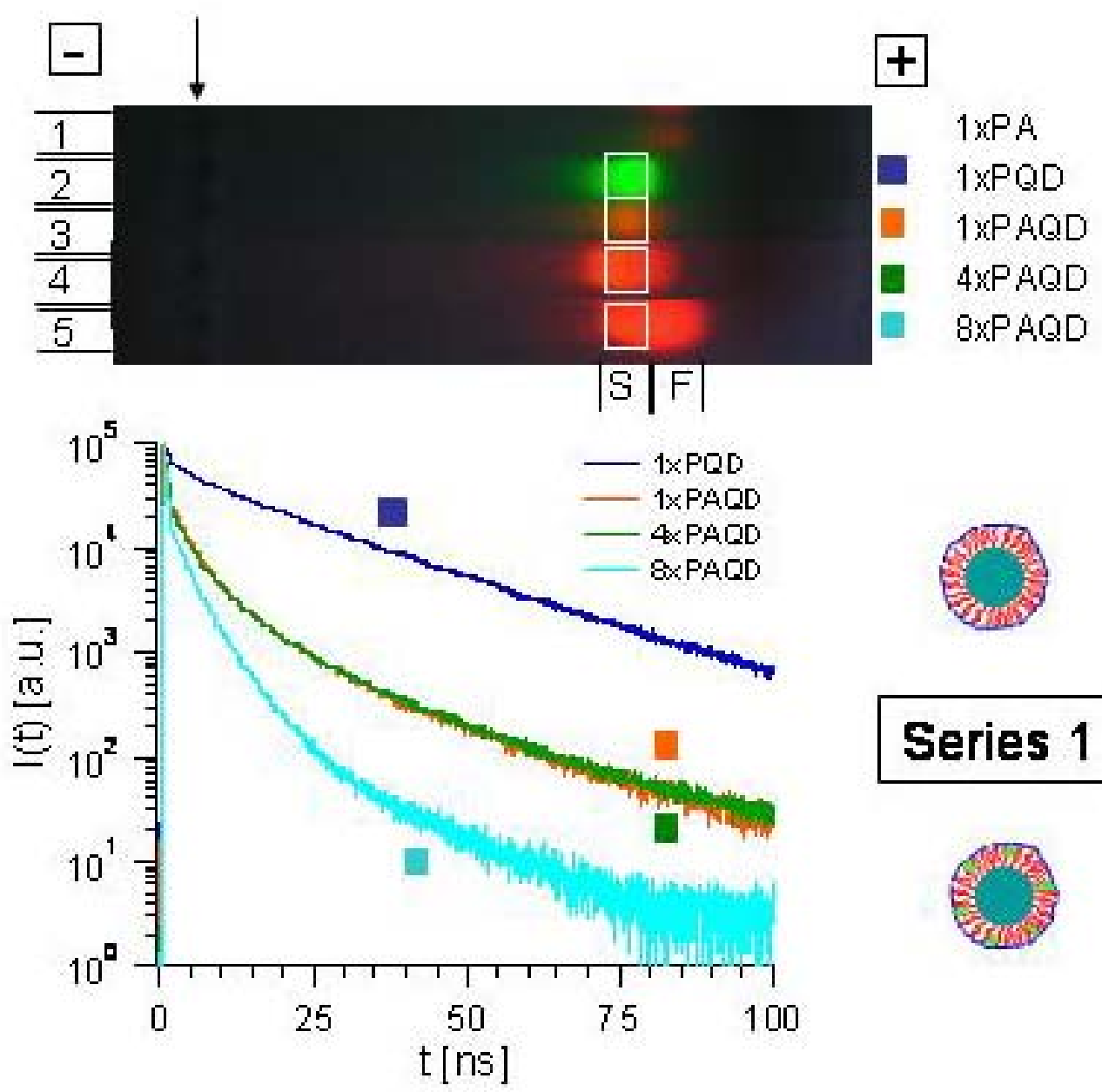

Series 1

Figure SI-VI.1. Fluorescence decay spectra of the samples shown in Figures SI-III.2 / SI-V.1. The corresponding decay times are found in Table SI-VI.1.

\begin{tabular}{l|l|l|l|l|}
\hline $\begin{array}{l}\text { Lifetime } \\
\text { decay } \\
\text { parameters }\end{array}$ & 1 xPQD & 1xPAQD & 4xPAQD & 8xPAQD \\
\hline$\tau_{1}\left(\mathrm{a}_{1}\right)$ & $0.23(0.23)$ & $0.31(0.30)$ & $0.23(0.32)$ & $0.3(0.39)$ \\
$\tau_{2}\left(\mathrm{a}_{2}\right)$ & $0.79(0.14)$ & $0.95(0.30)$ & $0.83(0.29)$ & $0.92(0.25)$ \\
$\tau_{3}\left(\mathrm{a}_{3}\right)$ & $2.06(0.10)$ & $2.66(0.23)$ & $2.50(0.25)$ & $2.38(0.17)$ \\
$\tau_{4}\left(\mathrm{a}_{4}\right)$ & $8.03(0.13)$ & $6.33(0.13)$ & $6.24(0.11)$ & $4.13(0.17)$ \\
$\tau_{5}\left(\mathrm{a}_{5}\right)$ & $20.77(0.40)$ & $16.10(0.04)$ & $16.88(0.03)$ & $7.13(0.02)$ \\
\hline$<\tau>$ & 10.09 & 2.51 & 2.14 & 1.65 \\
\hline
\end{tabular}

Table SI-VI.1: Fluorescence lifetimes for polymer-coated nanocrystals QD545-ITK with ATTO590 incorporated in the polymer coat. The raw data are shown in Figure SI-VI.1. $a_{i}$ is the fractional amplitude, $\tau_{i}$ the lifetime of the ith component of fluorescence decay; $\langle\tau\rangle$ is the weighted average decay time. All lifetimes are in $n$ s. 


\section{VI.3. Polymer-coated quantum dots with ATTO dye attached in post modification}
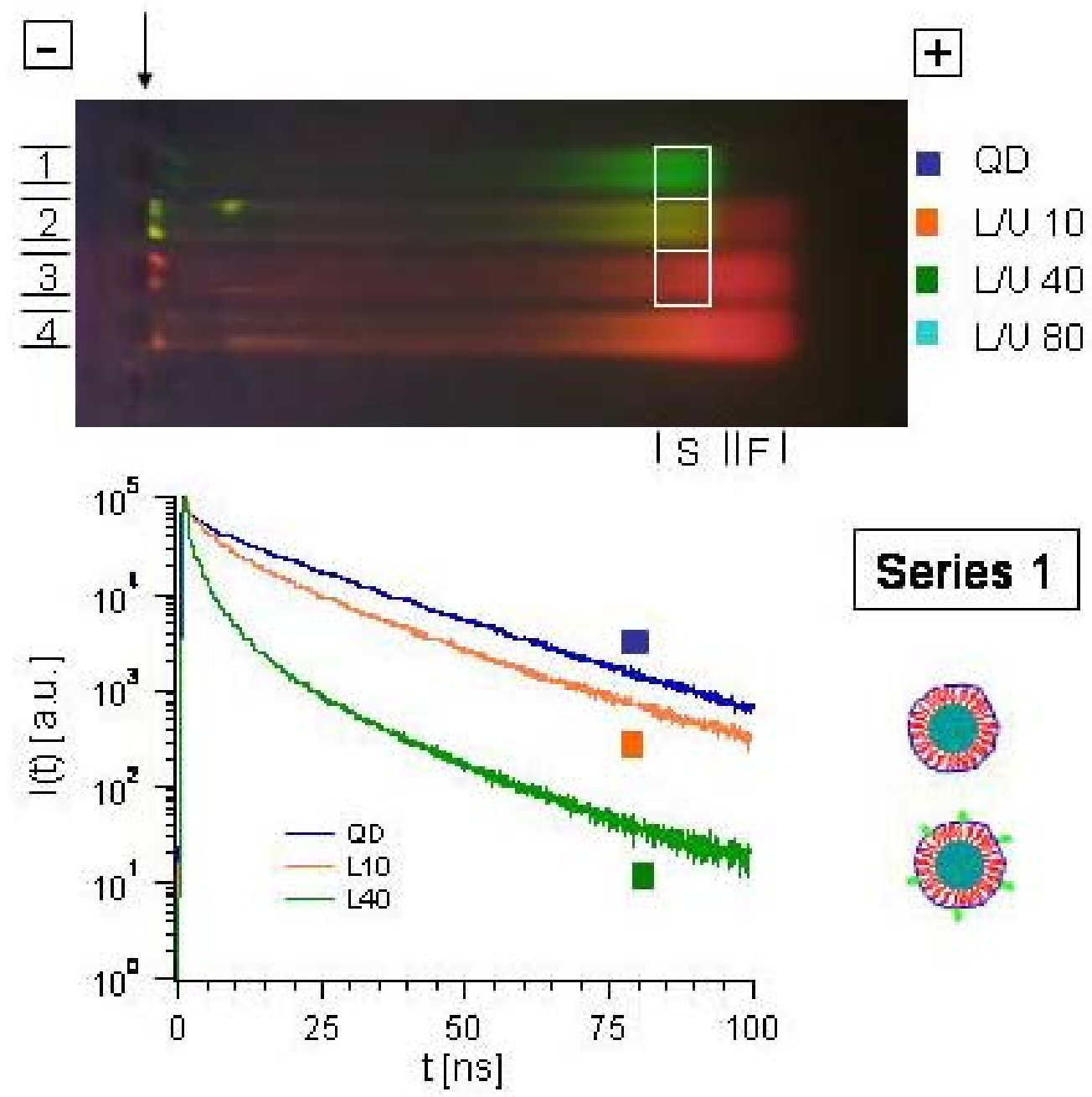

\section{Series 1}

Figure SI-VI.2. Fluorescence decay spectra of the samples shown in Figures SI-III.7 / SI-V.4. The corresponding decay times are listed in Table SI-VI.2.

\begin{tabular}{l|l|l|l|l|l|}
\hline $\begin{array}{l}\text { Lifetime } \\
\text { decay } \\
\text { parameters }\end{array}$ & QD & L10 & L40 & L80 & L160 \\
\hline$\tau_{1}(\mathrm{a} 1)$ & $0.23(0.23)$ & $0.40(0.25)$ & $0.32(0.40)$ & $0.38(0.41)$ & $0.39(0.45)$ \\
$\tau_{2}(\mathrm{a} 2)$ & $0.79(0.14)$ & $1.39(0.18)$ & $0.88(0.29)$ & $1.07(0.24)$ & $1.11(0.25)$ \\
$\tau_{3}(\mathrm{a} 3)$ & $2.06(0.10)$ & $4.63(0.27)$ & $2.10(0.19)$ & $2.57(0.19)$ & $2.34(0.18)$ \\
$\tau_{4}(\mathrm{a} 4)$ & $8.03(0.13)$ & $14.90(0.25)$ & $4.70(0.09)$ & $5.19(0.11)$ & $4.60(0.10)$ \\
$\tau_{5}(\mathrm{a} 5)$ & $20.77(0.40)$ & $29.65(0.05)$ & $11.65(0.03)$ & $12.55(0.05)$ & $10.7(0.02)$ \\
\hline$<\tau>$ & 10.09 & 6.91 & 1.62 & 2.32 & 1.66 \\
\hline
\end{tabular}

Table SI-VI.2: Compiled time resolved values for polymer-coated quantum dots with dye linked to the polymer-shell with a post-modification process. $a_{i}$ is the amplitude of the $\tau_{i}$ component of fluorescence decay; $\langle\tau>$ is the weighted average decay times. All time values are in $n$. The raw data are shown in Figures SI-VI.2 and SI-VI.3. 


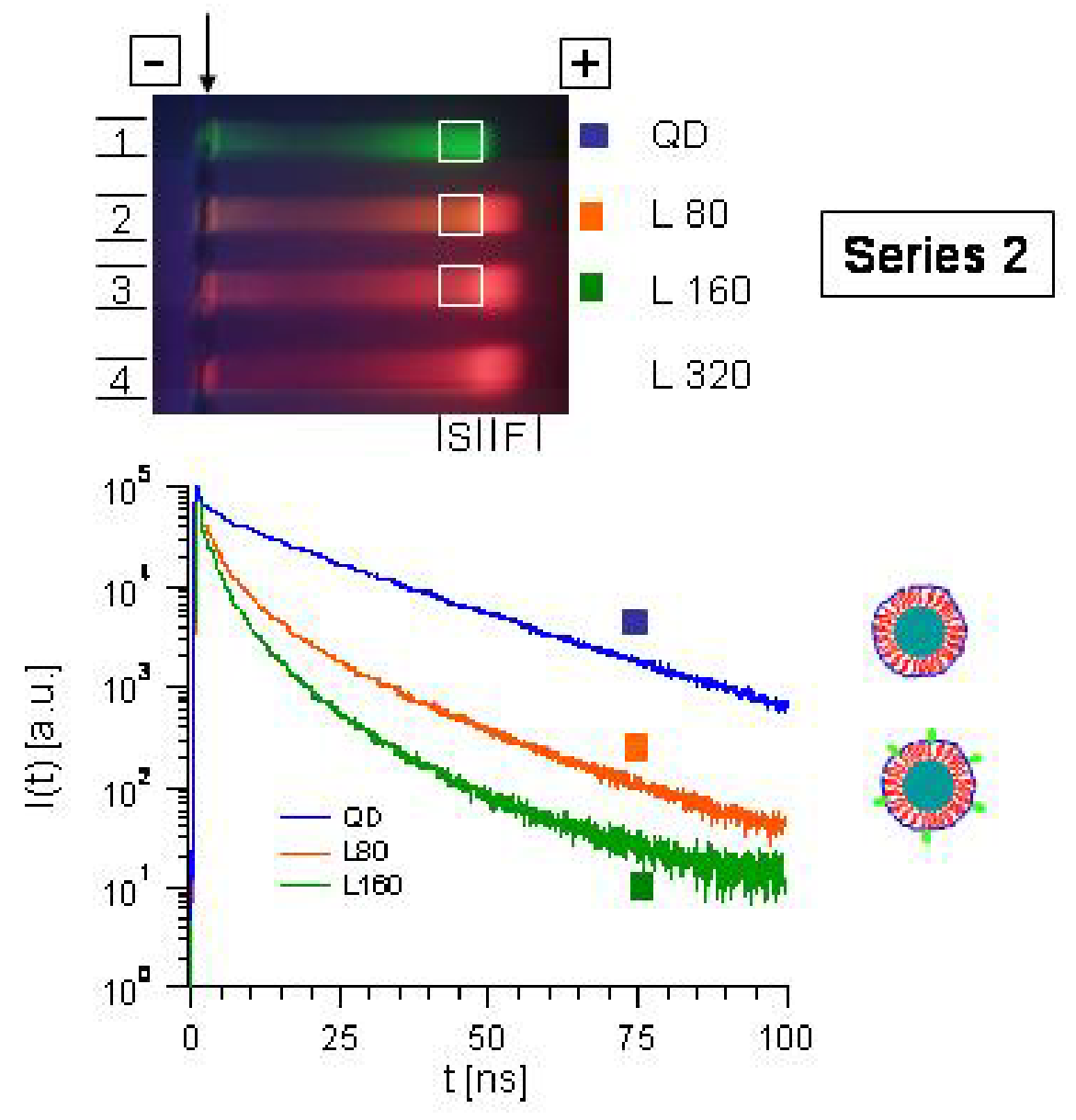

Fiqure SI-VI.3. Fluorescence decay spectra of the samples shown in Figures SI-III.9 / SI-V.5. The corresponding decay times are enlisted in Table SI-VI.2.

\section{V.4. Polymer-coated quantum dots with ATTO dye attached to a shell of PEG}

\begin{tabular}{l|l|l|}
\hline $\begin{array}{l}\text { Lifetime } \\
\text { decay } \\
\text { parameters }\end{array}$ & QD & L2 \\
\hline$\tau_{1}\left(\mathrm{a}_{1}\right)$ & $0.23(0.23)$ & $1.19(0.29)$ \\
$\tau_{2}\left(\mathrm{a}_{2}\right)$ & $0.79(0.14)$ & $3.29(0.23)$ \\
$\tau_{3}\left(\mathrm{a}_{3}\right)$ & $2.06(0.10)$ & $7.13(0.26)$ \\
$\tau_{4}\left(\mathrm{a}_{4}\right)$ & $8.03(0.13)$ & $16.89(0.20)$ \\
$\tau_{5}\left(\mathrm{a}_{5}\right)$ & $20.77(0.40)$ & - \\
$<\tau>$ & 10.09 & 7.10 \\
\hline
\end{tabular}

Table SI-VI.3: Compiled time resolved values for polymer-coated PEG-modified quantum dots with dye linked to the PEG-surface. $a_{i}$ is the amplitude of the $\tau_{i}$ component of fluorescence decay; $\langle\tau\rangle$ is the weighted average decay times. All time values are in ns. The raw data are shown in Figure SI-VI.4. 


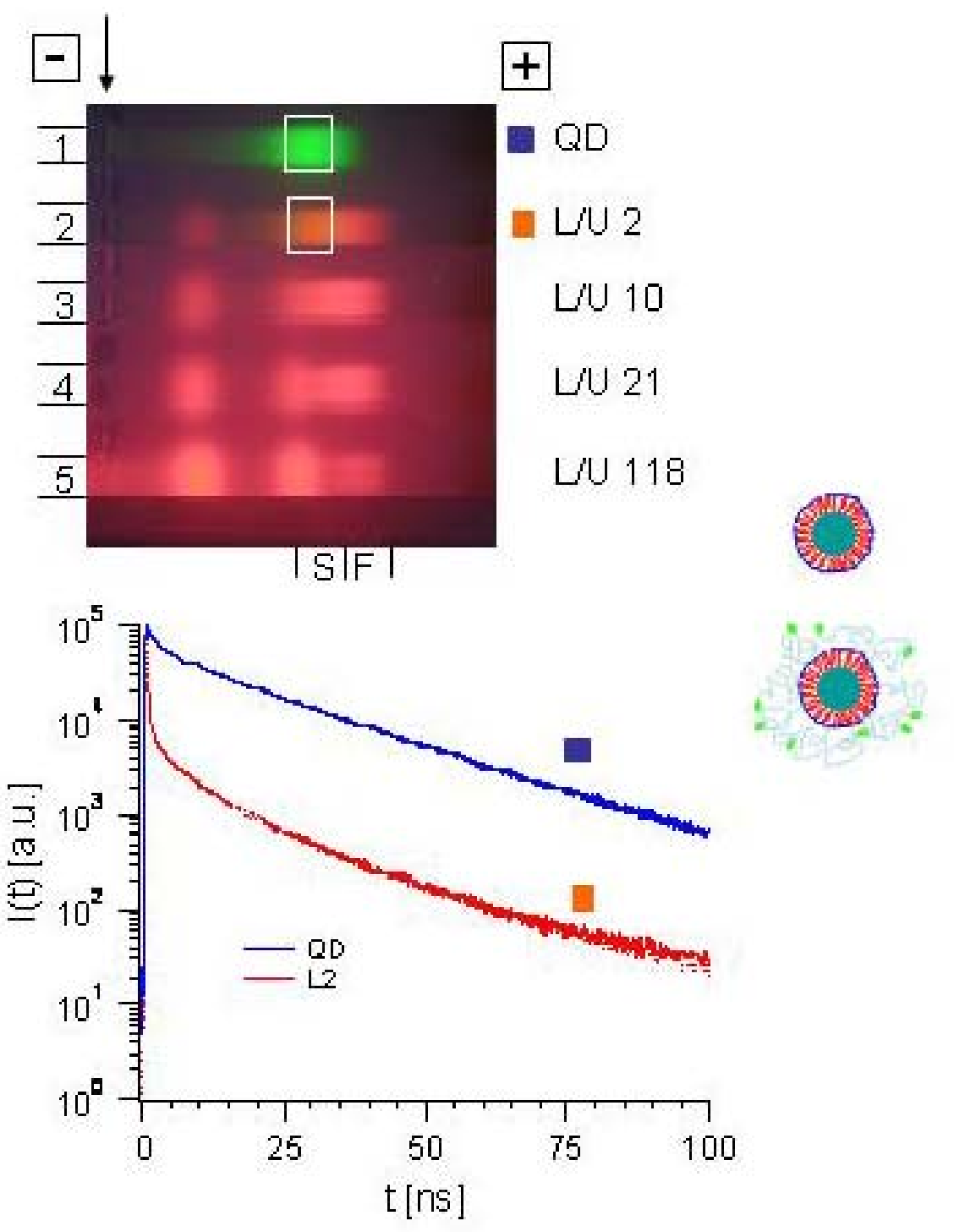

Figure SI-VI.4. Fluorescence decay spectra of the samples shown in Figures SI-III.11 / SI-V.6. The derived lifetimes are enlisted in Table SI-VI.3. 


\section{References}

1. Lin, C.-A. J.; Sperling, R. C.; Li, J. K.; AnitaTing-Ya; Zanelly, M.; Chang, W. H.; Parak, W. J. SMALL 2007, in preparation.

2. $\quad$ Pellegrino, T.; Manna, L.; Kudera, S.; Liedl, T.; Koktysh, D.; Rogach, A. L.; Keller, S.; Rädler, J.; Natile, G.; Parak, W. J. Nanoletters 2004, 4, (4), 703-707.

3. $\quad$ Sperling, R. A.; Pellegrino, T.; Li, J. K.; Chang, W. H.; Parak, W. J. Advanced Functional Materials 2006, 16, 943-948.

4. $\quad Y u, W . W . ; Q u, L . ;$ Guo, W.; Peng, X. Chemistry of Materials 2003, 15, (14), 2854-2860.

5. $\quad$ Sperling, R. A.; Liedl, T.; Duhr, S.; Kudera, S.; Zanella, M.; Lin, C.-A. J.; Chang, W.; Braun, D.; Parak, W. J. Journal of Physical Chemistry C 2007, accepted.

6. Dulkeith, E.; Ringler, M.; Klar, T. A.; Feldmann, J.; Javier, A. M.; Parak, W. J. Nanoletters 2005, 5, (4), 585-589.

7. Brochon, J. C., Maximum-Entropy Method Of Data-Analysis In Time-Resolved Spectroscopy. In Numerical Computer Methods, Pt B, 1994; Vol. 240, pp 262311.

8. Livesey, A. K.; Brochon, J. C. Biophysical Journal 1987, 52, (5), 693-706. 